\title{
The long non-coding RNA Dali is an epigenetic regulator of neural differentiation
}

\author{
Vladislava Chalei ${ }^{1}$, Stephen N Sansom ${ }^{1,2}$, Lesheng Kong ${ }^{1}$, Sheena Lee ${ }^{3}$, \\ Juan F Montiel ${ }^{1}$, Keith W Vance ${ }^{1 * \dagger \neq}$, Chris P Ponting ${ }^{1 * \dagger}$
}

${ }^{1}$ MRC Functional Genomics Unit, Department of Physiology, Anatomy and Genetics, University of Oxford, Oxford, United Kingdom; ${ }^{2}$ Computational Genomics Analysis and Training Programme, University of Oxford, Oxford, United Kingdom; ${ }^{3}$ Department of Physiology, Anatomy and Genetics, University of Oxford, Oxford, United Kingdom

*For correspondence: k.w.vance@bath.ac.uk (KWV); Chris.Ponting@dpag.ox.ac.uk (CPP)

tThese authors contributed equally to this work

Present address: ${ }^{\circ}$ Department of Biology and Biochemistry, University of Bath, Bath, United Kingdom

Competing interests: See page 21

Funding: See page 21

Received: 28 August 2014

Accepted: 21 November 2014

Published: 21 November 2014

Reviewing editor: Thomas $\mathrm{R}$ Gingeras, Cold Spring Harbor Laboratory, United States

(c) Copyright Chalei et al. This article is distributed under the terms of the Creative Commons Attribution License, which permits unrestricted use and redistribution provided that the original author and source are credited.
Abstract Many intergenic long noncoding RNA (IncRNA) loci regulate the expression of adjacent protein coding genes. Less clear is whether intergenic IncRNAs commonly regulate transcription by modulating chromatin at genomically distant loci. Here, we report both genomically local and distal RNA-dependent roles of Dali, a conserved central nervous system expressed intergenic IncRNA. Dali is transcribed downstream of the Pou3f3 transcription factor gene and its depletion disrupts the differentiation of neuroblastoma cells. Locally, Dali transcript regulates transcription of the Pou3f3 locus. Distally, it preferentially targets active promoters and regulates expression of neural differentiation genes, in part through physical association with the POU3F3 protein. Dali interacts with the DNMT1 DNA methyltransferase in mouse and human and regulates DNA methylation status of CpG island-associated promoters in trans. These results demonstrate, for the first time, that a single intergenic IncRNA controls the activity and methylation of genomically distal regulatory elements to modulate large-scale transcriptional programmes.

DOI: 10.7554/eLife. 04530.001

\section{Introduction}

A growing number of nuclear localised long noncoding RNAs (IncRNA, $\geq 200 \mathrm{nt}$ ) are known to regulate gene transcription and chromatin organisation (reviewed in (Vance and Ponting, 2014)). Many of these transcripts appear to act near to their site of synthesis to regulate the expression of genes locally on the same chromosome (cis-acting). Cis-acting IncRNA regulatory mechanisms have been described in detail for a number of enhancer associated nuclear IncRNAs, as well as IncRNAs involved in the processes of genomic imprinting and X chromosome inactivation (Tian et al., 2010; Melo et al., 2013; Monnier et al., 2013; Mousavi et al., 2013; Santoro et al., 2013; Vallot et al., 2013). Some cis-acting IncRNAs bind to DNA methyltransferase (DNMT) proteins and regulate genomic DNA methylation levels specifically at their sites of transcription (Mohammad et al., 2010; Di Ruscio et al., 2013).

Trans-acting IncRNAs that regulate gene expression across multiple chromosomes and on either allele have been documented less frequently. The ability of such IncRNAs to exert widespread effects on gene expression in trans is poorly understood, in large part because direct transcriptional targets for only very few of these transcripts have thus far been identified (Chu et al., 2011; Ng et al., 2013; Simon et al., 2011; Vance et al., 2014). Moreover, it is not clear whether these transcripts commonly act directly, or within ribonucleoprotein complexes, and how they might modify their target genes' regulatory landscape such as by regulating their DNA methylation profiles. 
eLife digest Traditionally genes are considered to contain all the instructions necessary to build proteins. For these instructions to be followed they need to be 'transcribed' into molecules called messenger RNA, which are then 'translated' to form the protein. Messenger RNAs are not the only type of RNA molecule made in a cell; long non-coding RNAs (or IncRNAs), for example, are transcribed but never translated into proteins. Instead, some IncRNAs control the expression of nearby genes and some alter how the DNA is packaged within the cell.

Several IncRNAs have been found to control their neighbouring genes, but it is unclear how many of these molecules can also regulate genes that are much further away, even on other chromosomes. One IncRNA called Dali is made in cells of the nervous system of mammals. In the genome, the gene for Dali is situated next to a gene called Pou3f3, which encodes a protein that contributes to the growth and development of nerves and the kidneys.

Chalei et al. have now shown that artificially reducing the amount of the Dali IncRNA restricts the development of mouse cells called N2A cells, which are commonly used to study the development of nerve cells. Reducing Dali IncRNA levels in these cells caused Pou3f3 messenger RNA levels to also decrease, which demonstrates that Dali is a IncRNA that controls its neighbouring gene. The levels of many other genes were also changed when Dali levels were reduced, including many genes that are needed to grow working nerve cells.

Chalei et al. also showed that the Dali IncRNA binds to 1427 different regions of the genome of N2A cells, most often near to the start of active genes; Dali could be carried to these sites by the POU3F3 protein. The DNA sequences with which the Dali IncRNA binds were all different. Chalei et al. found that Dali also binds to an enzyme, called DNMT1, that chemically modifies DNA to change how it is packaged into a cell, and they predict that this enzyme helps Dali to find its binding sites. Furthermore, when Dali IncRNA levels were artificially reduced, the chemical modifications that affect the packaging of DNA in the cell-and hence the expression of genes encoded by this DNA-were changed for several genes. Some of these genes were located far away from the gene that encodes Dali, indicating that this IncRNA can regulate the packaging and expression of distant genes.

Many genes that are regulated by Dali are also regulated by the POU3F3 protein; this suggests that the IncRNA might work together with this protein to affect the expression of some genes. Further work is now needed to uncover how many other IncRNAs act away from their sites of synthesis, and how many also form complexes with DNA-binding and DNA-modifying proteins.

DOI: 10.7554/eLife.04530.002

Many thousand mammalian intergenic IncRNAs have now been identified. Not all IncRNA transcript models will be functional, however. Single exon models, in particular, can be artefacts arising from genomic DNA contaminating sequencing libraries, and transcripts that are expressed at average levels lower than one copy per cell are less likely to confer function. Highly and broadly expressed, and bona fide monoexonic intergenic IncRNAs, such as Neat1 and Malat1/Neat2, however, appear not to have essential roles because their knockout mouse models are viable and fertile (Eissmann et al., 2012; Zhang et al., 2012). Transcript sequences and levels are thus not reliable predictors of mechanism. Instead, the significant temporal and spatial co-expression of genomically adjacent intergenic IncRNA and transcription factor genes might suggest that such IncRNAs commonly modulate transcriptional programmes that are initiated by these transcription factors (Ponjavic et alo, 2009). Indeed, several intergenic IncRNAs have well-documented cis-acting regulatory roles (Wang et al., 2011; Zhang et al., 2012; Berghoff et al., 2013).

Spatiotemporal co-expression of intergenic IncRNA and transcription factor genes is most pronounced during the development of the mouse central nervous system (CNS) (Ponjavic et al., 2009). To investigate the mechanistic basis of this physical linkage we chose to study a 3.5-kb, CNS-expressed, monoexonic, intergenic IncRNA termed Dali (DNMT1-Associated Long Intergenic), owing to its conservation of sequence and transcription across therian mammals and its genomic proximity to a transcription factor gene, Pou3f3 (also known as Brn1 or Oct8), which encodes a class III POU family transcription factor. Dali is transcribed in the sense orientation, relative to Pou $3 f 3$, from a locus $50 \mathrm{~kb}$ 
downstream of Pou3f3 within the flank of an extended genomic region (Figure 1A) that is characterised by near pervasive transcription in neuronal lineages (Ramos et al., 2013). Sauvageau et al. recently generated mouse knockout models for two of these intergenic IncRNA loci, linc-Brn1a, and linc-Brn1b (Figure 1A). Genomic deletion of the linc-Brn1b locus resulted in significant ( $50 \%)$ down-regulation of the upstream Pou3f3 gene, and linc-Brn $1 b^{-/}$mice exhibited abnormalities of cortical lamination and barrel cortex organization (Sauvageau et al., 2013). These abnormalities may derive from loss of the linc-Brn1b RNA transcript, or from the deletion of DNA functional elements (Bassett et al., 2014). The Dali locus is more distally located and does not overlap previously described IncRNA loci or regulatory elements (Figure 1A).

Pou3f3 is a single exon gene whose protein binds to DNA in a sequence-specific manner. Pou3f3 contributes to both neuronal and kidney development by regulating the proliferation and differentiation of progenitor cells (Nakai et al., 2003). Mouse mutants with homozygous loss of Pou3f3 die of renal failure within $36 \mathrm{hr}$ post partum (Nakai et al., 2003), with severe defects of the hippocampus and forebrain among others (McEvilly et al., 2002). In the developing neocortex, Pou3f3 is expressed in late neuronal precursors and in migrating neurons and, together with its closely related paralogue Pou3f2, is required in ventricular zone progenitors for deep-to-upper layer fate transition, sustained neurogenesis and cell migration (Dominguez et al., 2013).

Our experiments show that Dali is required for the normal differentiation of neural cells in culture. Furthermore, our results indicate that Dali functions by modulating the expression of its neighbouring Pou3f3 gene, as well as by interacting with the POU3F3 protein, and by directly binding and regulating the expression of genes involved in the neuronal differentiation programme in trans. Unexpectedly, Dali associates with the DNMT1 DNA methyltransferase and reduction of Dali levels increases DNA methylation at a subset of Dali-bound and -regulated promoters in trans. Our data therefore provide the first evidence that a IncRNA transcript can regulate multiple genes situated away from its site of synthesis by binding to promoter-proximal regulatory elements and altering their DNA methylation status in trans.

\section{Results}

\section{Conserved Dali genomic organisation and transcription}

Full-length mouse Dali is approximately $500 \mathrm{nt}(2.6 \mathrm{~kb})$ longer than a previously identified AK034039 cDNA cloned from the telencephalon (Figure 1-figure supplement 1A). Its locus, downstream of the Pou3f3 gene, contains mammalian conserved sequence both just upstream of its transcriptional start site, which presumably contributes to this locus' promoter, and within its transcribed sequence. A positionally equivalent and sequence-similar human DALI $(\sim 3.7 \mathrm{~kb})$ transcript was identified by RT-PCR and RACE in human foetal brain (Figure 1B; Figure 1-figure supplement 1B,C). Transcriptional evidence also exists for the orthologous locus in rat embryonic, as well as heart and kidney, samples (data not shown).

\section{Dali is a chromatin-associated transcript that is co-expressed with Pou3f3 in neural cell lineages}

ENCODE data indicate that both mouse and human Dali loci have the properties of a weak (or poised) enhancer in both brain and kidney tissues (Figure 1-figure supplement 1D,E). Consistent with this, Dali was most highly expressed in the adult brain and kidney, two of the three tissues displaying highest Pou3f3 expression, when profiled across a panel of adult mouse organs (Figure 1figure supplement 1F,G). In adult mouse (P56), Dali and Pou3f3 were expressed in all three regions of adult neurogenesis, the sub-ventricular zone (SVZ), olfactory bulb (OB), and dentate gyrus (DG) (Figure 1-figure supplement 1I) (Reviewed in Ming and Song, 2011). Dali was also co-expressed with Pou3f3 temporally and spatially in the developing mouse embryonic brain (Figure 1C,D). Both transcripts were up-regulated with the first appearance of cortical neurons (E10.5), and increased in expression further as the ratio between neurons and progenitors grew (Figure 1C,D). Furthermore, both Dali and Pou3f3 transcripts were undetectable in self-renewing mouse E14 embryonic stem (ES) cells, but after 3 days of retinoic acid (RA)-induced differentiation, a stage corresponding to the cell cycle exit of neuronal progenitors and their differentiation into neurons, these transcripts were rapidly up-regulated, their levels subsequently peaking at days 7 (Pou3f3) and 8 (Dali) (Figure 1E,F).

Mouse neuroblastoma N2A cells, which are frequently used as a neuronal progenitor-like cell type and an in vitro model of neuronal differentiation (Tremblay et al., 2010), express both Dali (at a 


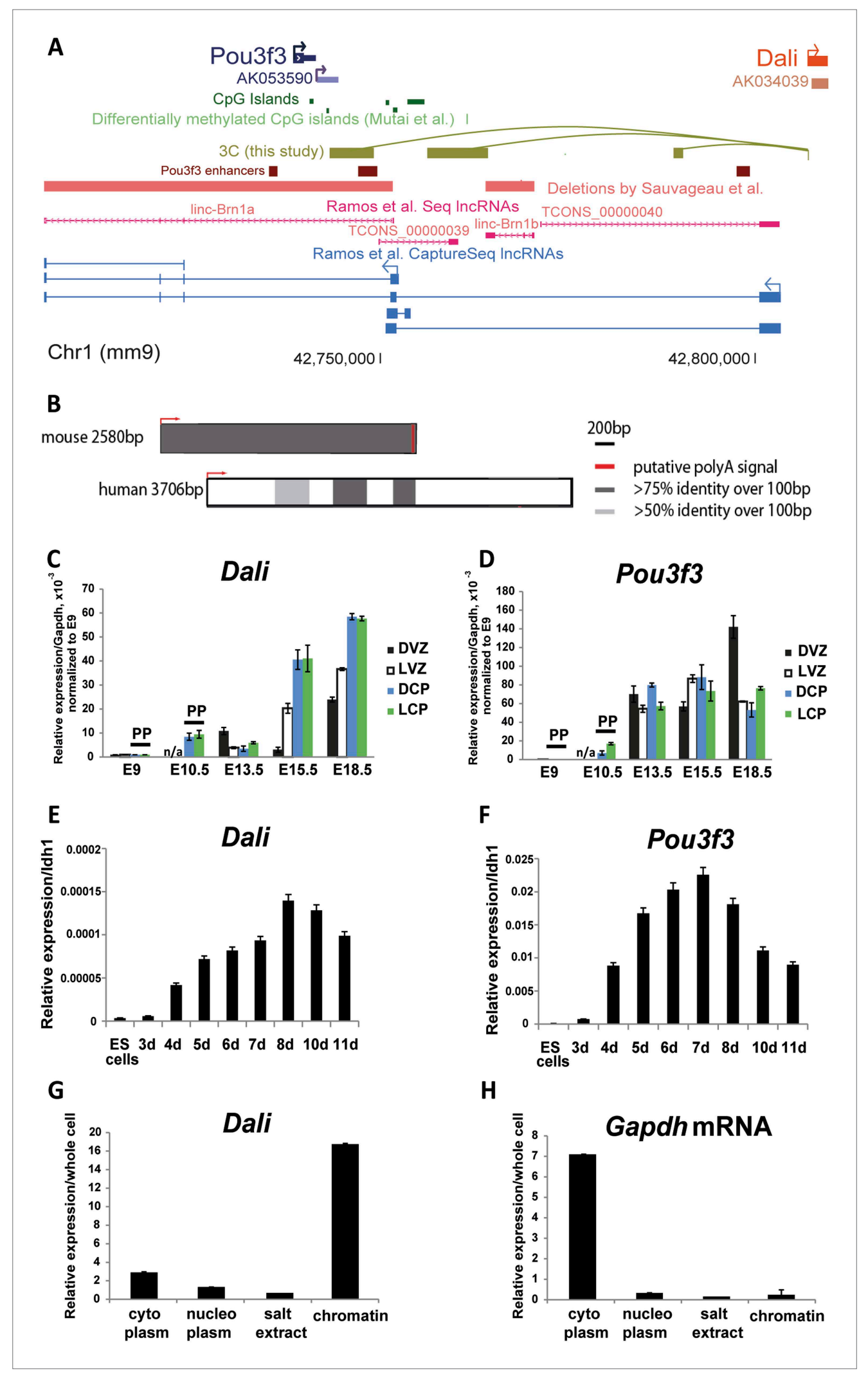

Figure 1. Conservation and expression within the Dali and Pou3f3 loci. (A) Schematic illustration of the mouse Pou3f3 genomic region showing coding and non-coding transcripts, enhancer elements from Vista Enhancer Browser (Visel et al., 2007), CpG islands, and published genomic deletions (Sauvageau et al., 2013). (B) Conservation and Figure 1. Continued on next page 
Figure 1. Continued

relative sizes of Dali transcripts in mouse and human confirmed by RACE. (C) Dali and (D) Pou3f3 are co-expressed temporally and spatially in the developing mouse brain. DVZ: Dorsal ventricular zone; LVZ: Lateral ventricular zone; DCP: Dorsal cortical plate; LCP: Lateral cortical plate; PP: pre-plate. The levels of Dali, Pou3f3 were measured by qRT-PCR. Results are normalised to Gapdh and presented relative to expression in E9.0 sample (set arbitrarily to 1). Mean \pm s.e., $n=3$ (technical replicates). (E and F) Similarly to Pou3f3, Dali is up-regulated during neuronal differentiation of mouse ES cells. Neuronal differentiation of mouse ES cells was induced using RA. The levels of Dali and Pou3f3 were measured by qRT-PCR. Results are presented relative to an Idh1 reference gene which does not change significantly during differentiation. Mean \pm s.e., $n=3$. ( $\mathbf{G}$ and $\mathbf{H})$ Dali is a chromatin associated transcript. The relative amounts of Dali $(\mathbf{G})$ and a control mRNA (Gapdh) $(\mathbf{H})$ in the indicated fractions were measured by qRT-PCR. Mean values \pm s.e. of three independent experiments.

DOI: 10.7554 /eLife. 04530.003

The following figure supplements are available for figure 1:

Figure supplement 1. Analysis of the mouse and human Dali loci. DOI: 10.7554/eLife.04530.004

Figure supplement 2. The Pou3f3 locus occurs in a folded nuclear conformation both prior to and after the onset of the expression of its transcripts.

DOI: 10.7554/eLife.04530.005

population-average level of 2 copies per cell (Figure 1-figure supplement $1 K)$ ) and Pou3f3. When first detected in neuronal-progenitor-dominated areas of the developing brain (E10.5), Dali is expressed at a level at least two orders of magnitude higher than in N2A cells (Figure 1-figure supplement 1H). However, in N2A cells treated with RA for $72 \mathrm{hr}$, Dali is up-regulated approximately 4.5-fold, similar to the up-regulation observed in embryonic cortical plate (both dorsal and lateral) between days E10.5 to E18.5 (Figure 1-figure supplement 1H). Therefore, despite Dali expression level differences in N2A cells and the in vivo system, N2A cells represent an appropriate model system in which to study Dali function. Furthermore, Dali, but not a control mRNA (Gapdh), was highly enriched in the nucleus of N2A cells, most abundantly in the chromatin fraction (Figure $1 G, H$ ). Taken together, the data suggest that Dali may be involved in regulating nuclear function during neuronal development, potentially in coordination with Pou3f3.

\section{Dali regulates neural differentiation of N2A cells}

We next investigated whether Dali regulates neural differentiation by generating three independent stable Dali knockdown N2A cell lines each showing approximately 50-70\% reduction of Dali transcript levels and inducing neural differentiation using RA (Figure 2A). Compared to a stable non-targeting control line, fewer differentiated cells of Dali knockdown lines developed neurites. Those that did exhibited shorter neurites, often with multiple short outgrowths emanating from the same cell, compared to one or two long neurites developed by differentiated control cells (Figure $2 B, C$ ) indicating that Dali is required for normal differentiation of N2A cells.

\section{Dali regulates neural gene expression}

To investigate the molecular function of Dali, we performed microarray analysis to profile the transcriptome of N2A cells in which Dali transcript levels had been depleted by $\sim 70 \%$ using transient transfection of a specific Dali targeting shRNA expression vector (Figure 2D; shRNA and RT-qPCR oligo sequences and positions can be found in Supplementary file 1). Dali knockdown resulted in statistically significant changes in expression levels for 270 genes (False Discovery Rate [FDR] < 10\%) compared to a non-targeting control (Supplementary file 2; Figure 2E). 14 of 15 of these genes were also determined as being differentially expressed, with similar fold changes, using RT-qPCR and two additional independent shRNA expression constructs targeting Dali (Figure 2-figure supplement 1C). Gene expression changes we observed using microarrays were thus unlikely to be dominated by offtarget effects of the shRNA used. Gene Ontology (GO) analysis revealed that Dali-regulated genes were significantly enriched in cell cycle, DNA repair, cellular response to stimulus, and cell projection assembly functions (Figure 2F and Supplementary file 2; Benjamini-Hochberg corrected $p \leq 0.05$ ). Taken together, these expression and loss of function studies suggest that Dali acts as a pro-differentiation factor in neural development. 
A

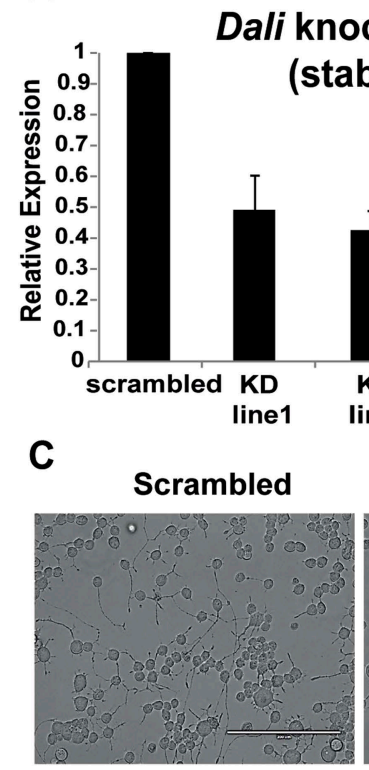

D

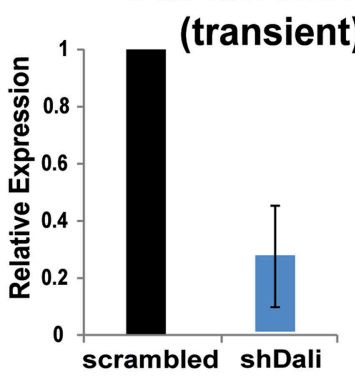

G
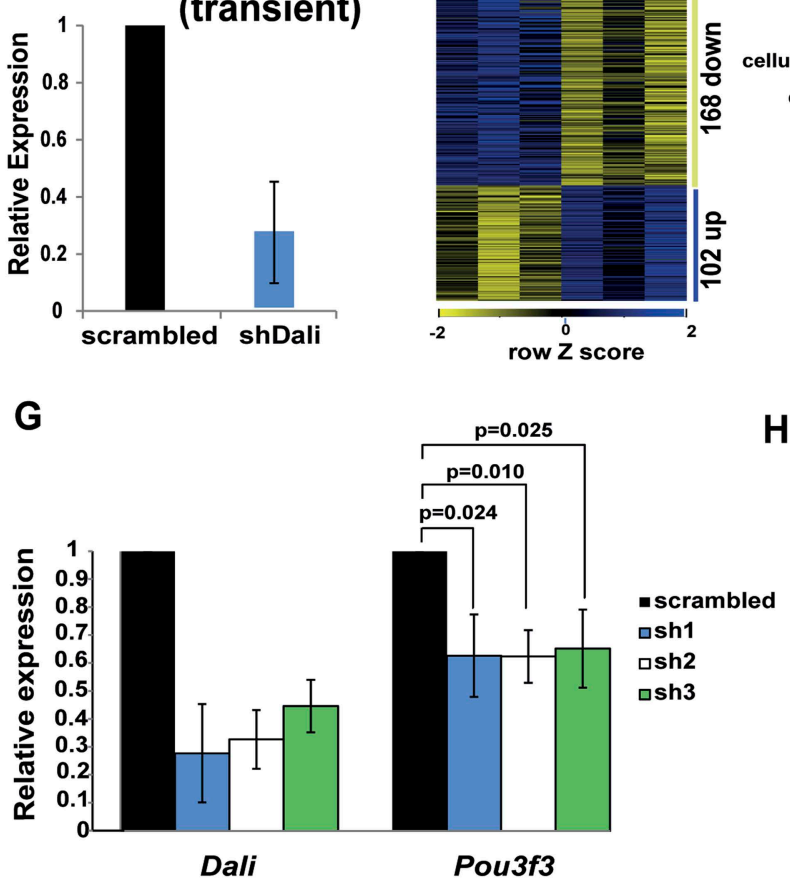

B

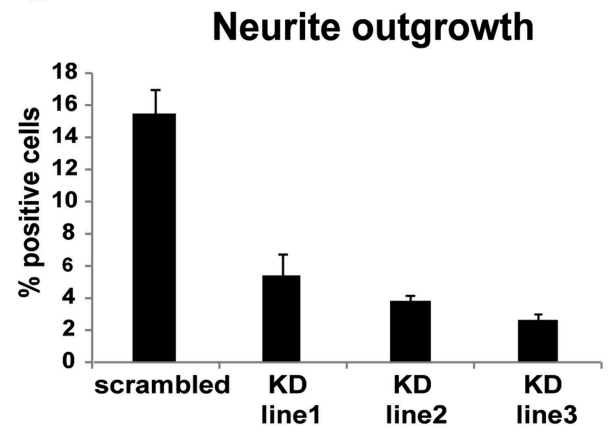

KD line2
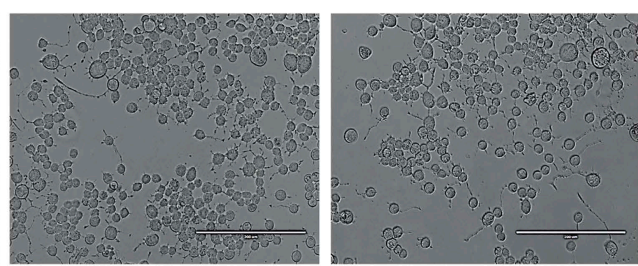

$\mathbf{F}$
E

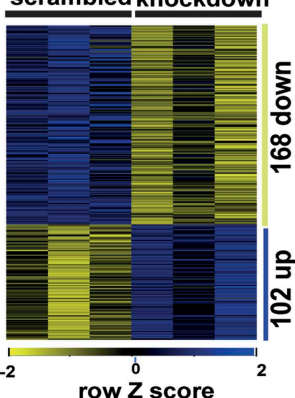

H

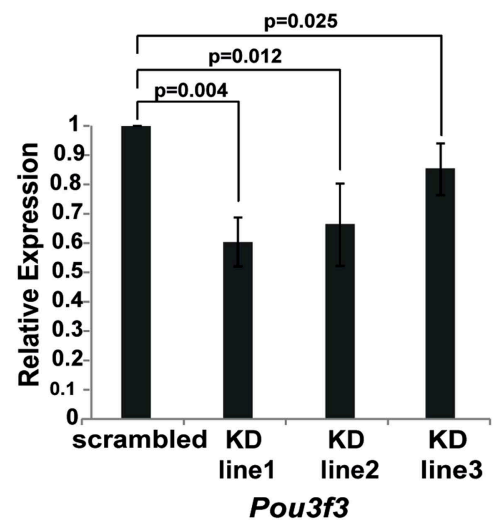

Figure 2. Dali plays a role in regulating genes in neuronal cells. (A) qRT-PCR analysis validates reduced levels of Dali in three clonal Dali knockdown cell lines compared to a control line. Mean values \pm s.e., $n=3$. (B) Reduced neurite outgrowth in RA-differentiated Dali knockdown cells. Cells were imaged using bright field microscopy. Cells with $\geq 1$ neurites of length greater than twice the cell body diameter were scored as positive. Average values \pm s.e., $n=3.500-600$ cells were counted in each case across at least three non-overlapping fields. (C) Representative images of control and stable Dali knockdown cells differentiated with RA for $72 \mathrm{hr}$. Scale bar $=200 \mu \mathrm{m}$. (D) N2A cells were transfected with either a non-targeting control (scrambled) or a Dali targeting shRNA expression vector

Figure 2. Continued on next page 
Figure 2. Continued

(shDali) for 72 hr. Mean values \pm s.e., $n=3$. (E) Transient Dali knockdown induces statistically significant changes in the expression of 270 genes in N2A cells (10\% FDR) (Supplementary file 2). (F) Gene Ontology (GO) categories significantly enriched among Dali regulated genes (5\% FDR, hypergeometric test, Benjamini and Hochberg correction; Supplementary file 2). (G) Decreased Pou3f3 expression upon Dali knockdown. Normalised using Gapdh, shown relative to a non-targeting control (set at 1). Mean values \pm s.e., $n=3$, one tailed t-Test, unequal variance. (H) Reduced Pou3f3 levels in stable Dali knockdown cells (see panel A). qRT-PCR results were normalised using Gapdh and presented relative to expression in control cells (set arbitrarily to 1). Mean values \pm s.e., $n=3$, one tailed t-Test, unequal variance.

DOI: 10.7554/eLife.04530.006

The following figure supplement is available for figure 2:

Figure supplement 1. Non-coding transcripts in the Pou3f3 locus form a network of regulatory interactions. DOI: 10.7554/eLife.04530.007

\section{Dali and Pou3f3 share transcriptional targets}

To investigate whether Dali knockdown affects expression of the adjacent Pou $3 \mathrm{f3}$ gene, we reduced its levels by transient transfection of three different shRNA constructs in N2A cells. After 72 hr, reduction of Dali levels by an average of $60-70 \%$ was found to reduce Pou3f3 transcript levels by approximately 40\% (Figure 2G). Three independent stable Dali knockdown clones in which Dali levels were reduced by $50-60 \%$ (Figure 2A) also showed $\sim 15-40 \%$ lower Pou3f3 levels (Figure 2 H). This suggests that the Dali transcript positively regulates Pou3f3 expression in an RNA-dependent manner. The genome-wide transcriptional response to Dali knockdown thus could be explained, in part, by its effect on Pou3f3.

Levels of another transcript, AK011913, expressed downstream of Pou3f3 (Figure 1A) were reduced by approximately 55\% upon Dali knockdown (Figure 2-figure supplement 1A). Reduction of AK011913 levels by approximately 60\% using shRNAs resulted in Dali and Pou3f3 levels decreasing by $73 \%$ and $82 \%$, respectively (Figure 2-figure supplement 1B). Linc-Brn1a, a IncRNA upstream of and sharing a bi-directional promoter with Pou3f3, was up-regulated by approximately $90 \%$ upon AK011913 depletion (Figure 2-figure supplement 1B). This is reminiscent of the down-regulation of Pou $3 f 3$ and up-regulation of lincBrn-1a following knockdown of another IncRNA downstream of Pou3f3, lincBrn-1b (Figure 1A) (Sauvageau et al., 2013). Together with previous reports, our data show the opposing regulatory influences of IncRNAs transcribed up- and downstream of Pou3f3 on its expression. Noncoding transcripts expressed from the extended Pou3f3 locus thus contribute to a complex network of regulatory interactions.

Furthermore, Chromatin Conformation Capture (3C) showed that the Dali promoter contacted three regions across the Pou3f3 locus (Figure 1A) in ES derived neuronal precursors (Figure 1-figure supplement 2) : 1) an enhancer element sequence lying upstream of Pou3f3 within the linc-Brn1a locus, 2) a region overlapping the $3^{\prime}$ UTR of Pou3f3 and full-length AK53590 (which are both regulated by Dali), as well as parts of TCONS_00000039 and linc-Brn1b, including a differentially methylated region reported to be important in regulating Pou3f3 expression (Mutai et al., 2009), and 3) a region lying within another non-coding locus (TCONS_00000040) (Ramos et al., 2013). Neither Dali nor Pou3f3 appears to play a role in initiating these DNA looping interactions because these contacts were present in E14 ES cells where neither is expressed (Figure 1-figure supplement 2B). Nevertheless, the Dali locus appears to contribute to an extended structurally and transcriptionally complex region centred on the Pou3f3 gene.

To examine to what extent the transcriptional response to Dali knockdown can be explained by its effect on Pou3f3, we reduced the level of Pou3f3 transcript in N2A cells by $35 \%$ using transient transfection of a Pou3f3 targeting shRNA vector (Figure 3A) and using microarrays observed statistically significant expression changes in 1041 genes (FDR <10\%; Figure 3B). Dali transcript levels do not change upon Pou3f3 depletion (Figure 3A). Genes differentially expressed after Pou3f3 knockdown were enriched in categories related to cell division and cell cycle (Figure 3C). The intersection between the sets of genes differentially expressed in Dali or in Pou3f3 knockdown cells was 6.2-fold greater than expected by chance ( $p$-value $<2.2 \times 10^{-16}$ ), and represented $31 \%$ of all genes differentially expressed in Dali knockdown cells (Figure 3D). Approximately equal numbers of genes shared between the two datasets were down- (43 genes) or up-regulated (41 genes) in both experiments (Supplementary file 3). A strong correlation was observed between the fold-change values of differentially expressed genes 
A

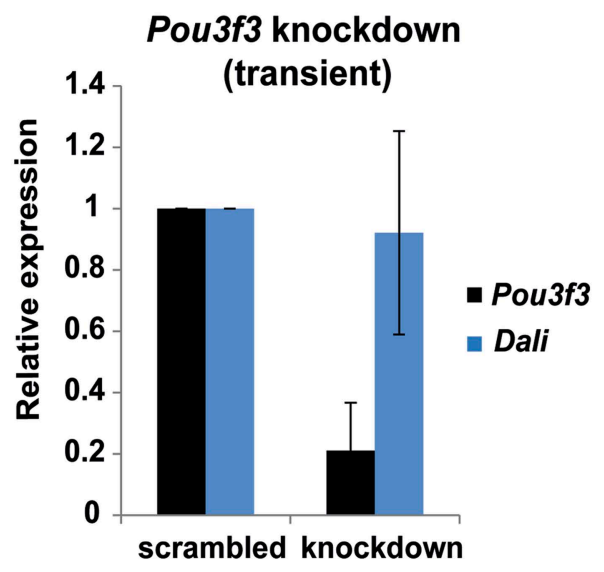

C

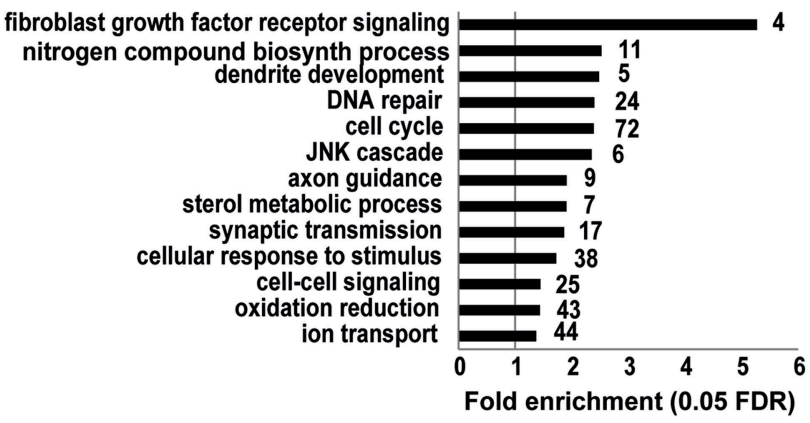

E

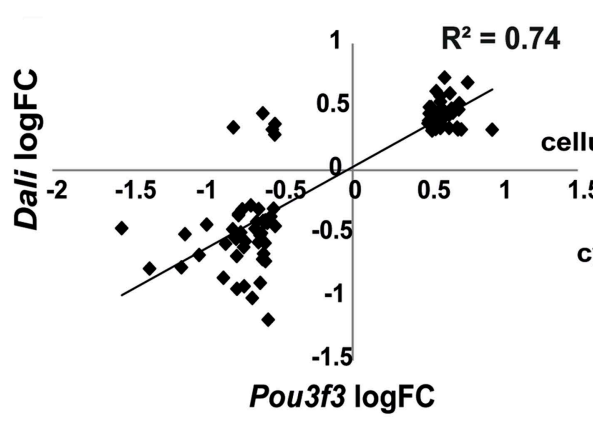

F
B

Pou3f3

scrambled knockdown

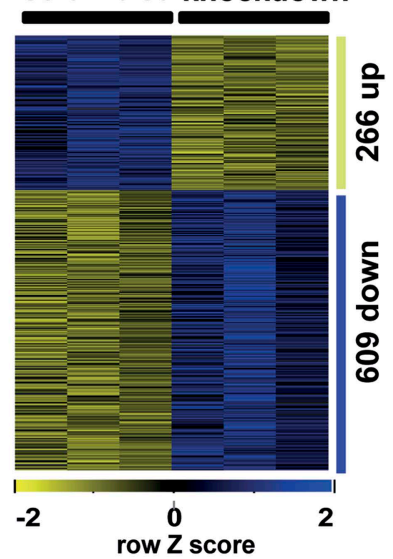

D

Dali knockdown

(0.1 FDR)

chromatin assembly

MAPKKK Cascad

DNA repair

cell cycle
cesponse to rimulus

cell cycle

cytoskeleton organization

dendrite development

axon guidance

sterol metabolic process

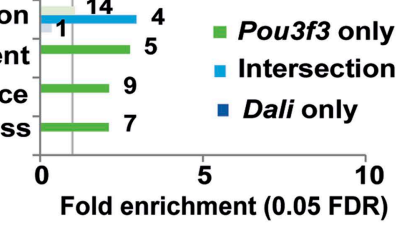

Figure 3. Dali regulates transcription in both Pou3f3-dependent and -independent manners. (A) N2A cells were transfected with either a non-targeting control (scrambled) or a Pou3f3 targeting shRNA expression vector (knockdown). Pou3f3 and Dali levels were measured by qRT-PCR $72 \mathrm{hr}$ post- transfection. Mean values \pm s.e., $\mathrm{n}=3$. (B) Pou3f3 knockdown resulted in statistically significant changes in the expression of 1041 genes in N2A cells ((10\% FDR, Supplementary file 3). (C) GO-analysis of genes differentially expressed upon Pou3f3 analysis (5\% FDR, hypergeometric test, Benjamini and Hochberg correction; Supplementary file 3). (D) Intersection of Pou3f3 and Dali targets shows a significant (Fisher's exact test) overlap approximately 6.2 times as large as expected by chance alone. (E) Target genes common between Dali and Pou3f3 show correlated expression, with the nearly all being positively or negatively regulated by both factors ( $R=0.74$; Supplementary file 3 ). (F) Enrichments of Gene Ontology categories of Pou3f3-dependent or -independent Dali targets.

DOI: 10.7554/eLife.04530.008 
in Dali and Pou3f3 knockdown experiments ( $R=0.74$; Figure 3E). Genes that were significantly differentially expressed only when Dali was depleted were enriched in chromatin assembly and MAPKKK signalling functions, whilst genes that were differentially expressed only when Pou3f3 transcripts were depleted were preferentially involved in dendrite development and axon guidance (Figure 3F). Cell cycle, DNA repair, and cellular response to stimulus genes were regulated by Dali in both Pou3f3dependent and -independent manners. We conclude that Dali and Pou3f3 interact, either genetically or molecularly, to regulate a subset of common targets involved in neural differentiation, and that Dali also likely possesses Pou3f3-independent transcriptional regulatory functions.

\section{Dali regulates gene expression programmes during neural differentiation of N2A cells}

To further investigate the role of Dali in neuronal differentiation we profiled the transcriptomes of proliferating or RA differentiated control and Dali stable knockdown N2A cell lines. In proliferating cells, 733 genes were differentially expressed between Dali knockdown and control cells (Figure 4A), including many genes with functions related to neuronal differentiation, apoptosis, neuronal function (Figure 4B). RA-mediated neuronal differentiation induced expression changes in 958 genes in control cells and 1016 genes in Dali knockdown cells (Figure 4-figure supplement 1A,B). Based on GO category annotations, differentiation of control or Dali knockdown cells was broadly similar, and was associated with altered expression of cell cycle, cell differentiation, energy metabolism, and neuron projection (Figure 4-figure supplement 1C,D). However, 804 genes were differentially expressed between terminally differentiated control and Dali knockdown cells (Figure 4C), of which 376 genes (46.8\%) also differed in expression between Dali knockdown and control cells prior to their differentiation (Figure 4E). The 428 genes that were significantly altered in expression only between stable Dali and control differentiated cells were enriched in functional categories relating to sterol biosynthesis, energy metabolism, cell cycle, response to chemical stimulus, cell cycle, adhesion and small GTPase signalling (Figure 4D). All 11 (of 34 known) sterol biosynthesis genes were down-regulated in Dali knockdown cells. This observation is consistent with the impaired neurite outgrowth of stable Dali knockdown cells because neuritogenesis and neurite outgrowth critically rely on membrane biosynthesis, and therefore, on expression of sterol biosynthesis genes (Paoletti et alo, 2011).

In addition, several key neuronal differentiation genes, for example Nrcam, Dscam, Dlx1 and Pax3, were differentially expressed between Dali knockdown and control cells both prior to and after differentiation. Furthermore, multifactorial analysis of RA-induced gene expression changes in control and stable Dali knockdown cells showed that 174 genes responded to RA differently depending on the presence or knockdown of Dali (FDR 5\%; Supplementary file 4). These were significantly enriched in categories relating to neuronal development (Figure 4F), including pro-differentiation factors such as the inhibitor of Wnt signaling Dkk1 (Cajanek et al., 2009) and Wnt receptor Fzd5 (Kemp et al., 2007).

In summary, compared to control cells, stable Dali knockdown cells exhibit contrasting alterations in gene expression programmes before and after RA-induced differentiation. In both cases, these programmes are enriched in functional categories related to neural differentiation and function, consistent with the proposed role for Dali in neural development.

\section{Dali preferentially binds to active promoters in trans}

We next sought to identify and characterise genes that are both bound and regulated by Dali. To do so, we determined the genome-wide binding profile of Dali in N2A cells using Capture Hybridisation Analysis of RNA Targets (CHART)-Seq (Simon et al., 2011; Simon, 2013) (Figure 5-figure supplement $1 \mathrm{~A}-\mathrm{C}$ ). We discovered 1427 focal Dali-associated regions genome-wide (Figure 5A,B; Supplementary file 5), of which all nine selected loci were validated by CHART-qPCR in an independent experiment (Figure 5-figure supplement 1D).

Dali binding sites were typically limited to less than $1 \mathrm{~kb}$ in length (Figure 5-figure supplement 1E) and were distributed across the genome with no apparent chromosomal biases other than a depletion on the $\mathrm{X}$ chromosome which may reflect the inactivation of one $\mathrm{X}$ chromosome copy in these female N2A cells (Figure $5 \mathrm{C}$ ). These sites were preferentially located at the $5^{\prime}$ end of protein coding genes (Figure 5D): $30.5 \%$ of peaks were within $5 \mathrm{~kb}$ of a transcriptional start site (TSS) (Figure 5E). Dali bound sequences were significantly enriched for $\mathrm{H} 3 \mathrm{~K} 4 \mathrm{me} 3, \mathrm{H} 3 \mathrm{~K} 4 \mathrm{me} 1$ and $\mathrm{H} 3 \mathrm{~K} 27 \mathrm{ac}$ modified histones and Polll occupancy, and were depleted for repressive histone marks (Figure 5F). This suggests that Dali preferentially associates with regions of active chromatin. GO category enrichment analysis 
A

\section{Undifferentiated cells}

Dali

scrambled knockdown

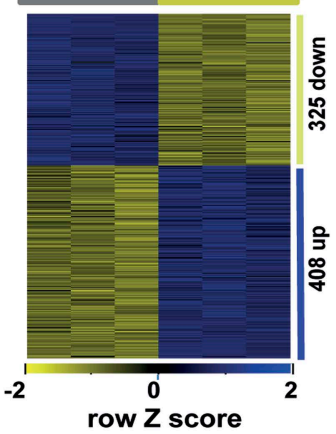

C

\section{RA differentiated cells}

Dali

scrambled knockdown

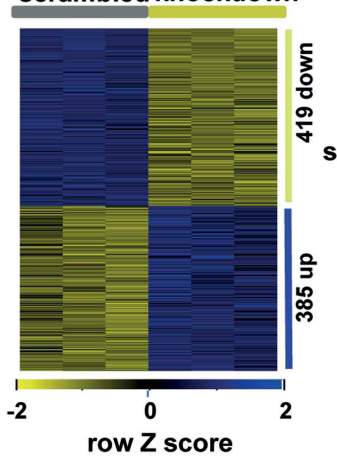

E

Undifferentiated cells
B

\section{Undifferentiated cells: stable Dali KD vs control}

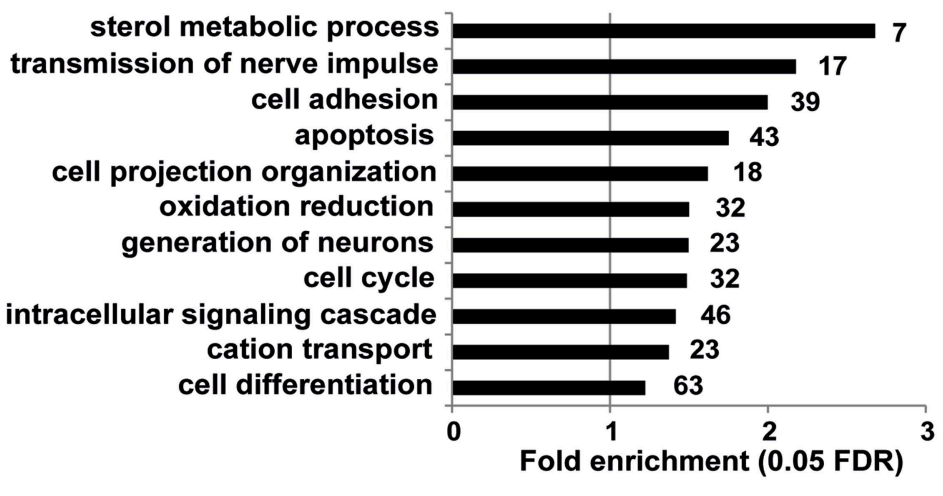

D

RA differentiated cells: stable Dali KD vs control sterol biosynthetic process axon extension cellular alcohol metabolic process oxidation reduction small GTPase mediated signal transduction 19 carbohydrate metabolic process 11 response to chemical stimulus 19 cell adhesion 15 cell cycle 16 ion transport 14

$\begin{array}{lllll}01 & 5 & 10 & 15 & 20\end{array}$

Fold enrichment (0.05 FDR)

$\mathbf{F}$

"Differential responders"

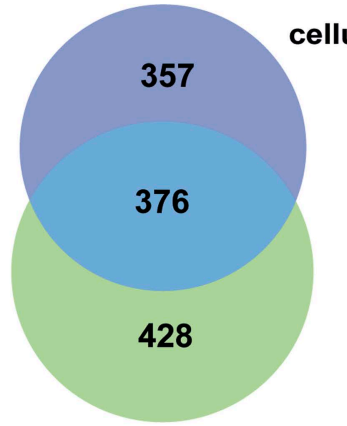

RA differentiated cells

ellular carbohydrate metabolic process 5

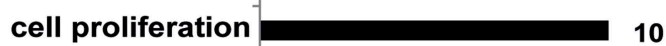

response to chemical stimulus 11

neurogenesis $\quad 7$

nervous system development 11

cell differentiation 19

regulation of transcription 19

cell communication

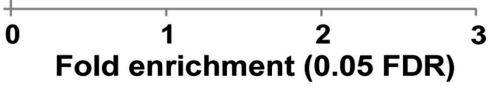

Figure 4. Gene expression analysis of stable Dali knockdown cells. (A) Stable Dali knockdown resulted in statistically significant changes in the expression of 747 genes in N2A cells (1.3-fold, 5\% FDR, Supplementary file 4). 332 genes were up-regulated, 415 down-regulated. (B) GO-analysis of genes differentially expressed upon stable Dali depletion (5\% FDR, hypergeometric test, Benjamini and Hochberg correction). (C) Stable Dali knockdown and control cells were differentiated with retinoic acid for $72 \mathrm{hr}$. 825 genes were differentially expressed between differentiated knockdown and control lines (( $\geq 1.3$-fold, 5\% FDR, Supplementary file 4). 436 genes were up-regulated, 389 down-regulated. (D) GO-analysis of genes differentially expressed only between differentiated stable Dali knockdown and control cells (5\% FDR, hypergeometric test, Benjamini and Hochberg correction). (E) Intersection of gene sets differentially expressed between stable Dali knockdown and control cells prior to (undifferentiated) and after retinoic acid addition

Figure 4. Continued on next page 
Figure 4. Continued

(differentiated). (F) GO-analysis of genes responding to retinoic acid treatment differently between stable Dali knockdown and control cells (5\% FDR, hypergeometric test, Benjamini and Hochberg correction). 'Differential responder' genes were identified using multifactorial analysis of the stable Dali knockdown arrays using limma (Smyth, 2004).

DOI: 10.7554/eLife.04530.009

The following figure supplement is available for figure 4:

Figure supplement 1. Transcriptomics.

DOI: 10.7554/eLife.04530.010

showed that genes associated with Dali peaks contribute to processes related to neuronal differentiation (cell cycle), neuronal projection development (cytoskeleton organization and small GTPase mediated signal transduction), neuronal function (synaptic transmission), and more general cellular processes, such as gene expression, intracellular signalling, and cellular homeostasis (Figure 5G). 150 genes ( $8.6 \%$ of all Dali bound genes) regulated by Dali contained Dali binding sites within their regulatory regions (Figure $5 \mathrm{H}$ ) and presumably represent direct transcriptional targets.

\section{Dali interacts with chromatin modifying proteins}

To investigate the mechanisms of its genomic targeting, we next performed computational analysis of Dali bound sequences. We discovered that Dali binding sites do not exhibit significant sequence complementarity with the Dali transcript (Figure 5-figure supplement 1F, see Methods), and are not likely to form RNA-DNA:DNA triplex structures (Figure 5-figure supplement 1G), suggesting that Dali does not bind DNA directly. We therefore speculated that Dali may be targeted to the genome indirectly thorough RNA-protein interactions. To identify proteins that interact directly with Dali, we performed a pull down assay in which in vitro transcribed and $5^{\prime}$ end-biotinylated Dali was incubated with nuclear extract prepared from day 4 RA-differentiated ES cells. We identified, using mass spectrometry, 50 proteins that associated with Dali, but not with antisense Dali or a size-matched unrelated control transcript (Supplementary File 7). Direct interactions between the endogenous Dali transcript and four of these candidate binding proteins, the DNA methyltransferase DNMT1, the BRG1 core component of the SWI/SNF family chromatin remodelling BAF complex, and the P66beta, and SIN3A transcriptional co-factors, were subsequently validated using UV-crosslinked RNA Immunoprecipitation (UV-RIP) in N2A cells (Figure 6A,B). Human DALI was also found, using UV-RIP, to interact with human DNMT1, yet not with BRG1, in human neuroblastoma SH-SY5Y cells (Figure 6B). Consequently, in further experiments, we focused on the evolutionarily conserved DNMT1 interaction.

Interestingly, 9 of 58 human transcription factors reported by Hervouet et al. as interacting with the DNMT1 protein (Hervouet et al., 2010), including CTCF, but also AP-2, C-ets-1, LRH1, PARP, PAX6, STAT1, YY1, and Sp1, were found to have binding site motifs that were significantly enriched within our stringent Dali bound CHART-seq peaks (Supplementary File 6). Motifs for none of 42 transcription factors that do not interact with DNMT1 but interact with DNMT3a and/or DNMT3b (Hervouet et al., 2010) were enriched in these peaks (Supplementary File 6). In particular, using a de novo motif discovery approach, we found a highly-enriched CTCF-binding site-like motif in 125 out of 1427 Dali peaks (9\%; MEME E-value = $3.1 \times 10^{-62}$; Figure 6C) (Supplementary File 7). This result was concordant with the greater than expected overlap between Dali-associated regions and known CTCF binding sites in neuronal tissues (Figure 5F) (Shen et al., 2012). Using Chromatin Immunoprecipitation and qPCR (ChIP-qPCR) in N2A cells, we confirmed the CTCF-enrichment of previously-known CTCFbinding sites within 7 Dali-bound and regulated promoters, but not at four control regions (Figure 6D). However, despite CTCF and Dali thus occupying a subset of shared genomic binding sites, UV-RIP provided no evidence of a direct physical interaction (Figure 6E). Consequently, Dali and CTCF may be non-interacting molecular subunits of a larger ribonucleoprotein complex, or alternatively they might independently bind adjacent sequence, or compete for binding to the same region. Taken together, the data suggest that Dali is recruited to chromatin via indirect interactions with several DNA-binding proteins through its direct association with DNMT1.

\section{Depletion of Dali leads to DNA methylation changes at bound and regulated promoters}

Increasing numbers of IncRNAs have been shown to direct DNA methylation changes at their sites of synthesis (Mohammad et al., 2010; Di Ruscio et al., 2013). The direct interaction of Dali with DNMT1, 
A

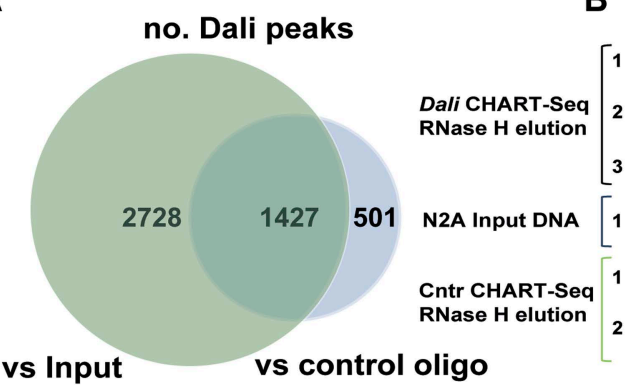

B Ache

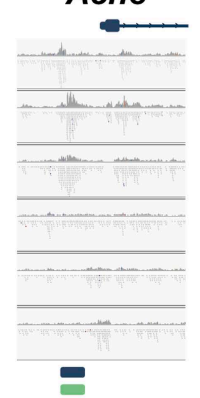

E2f2
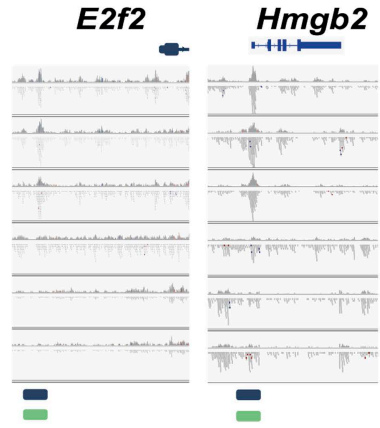

D
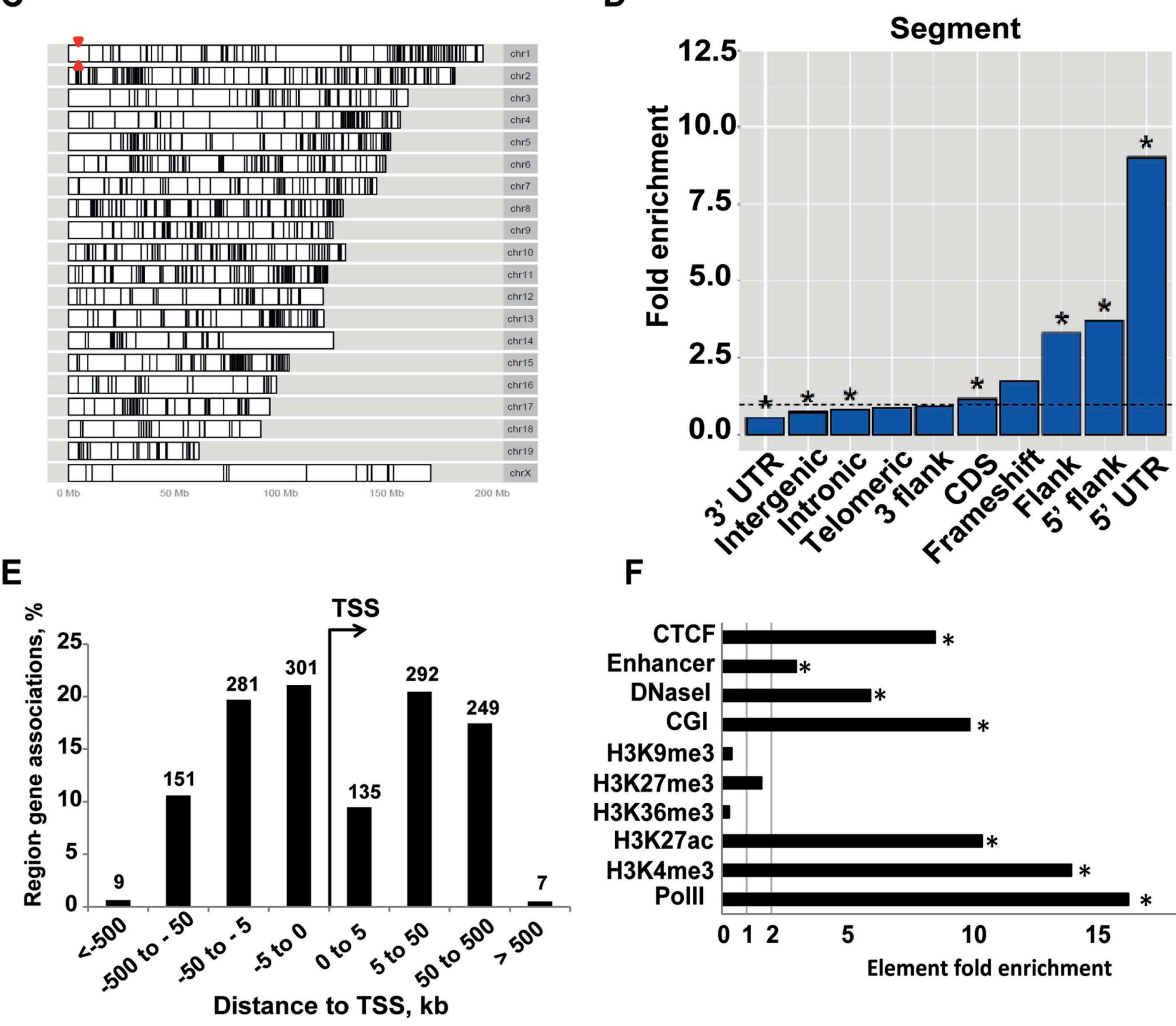

G

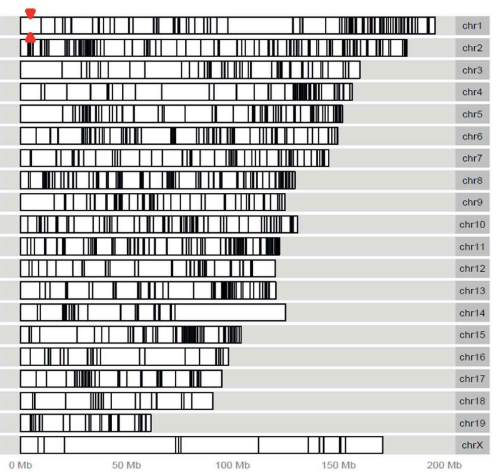

$\mathbf{F}$

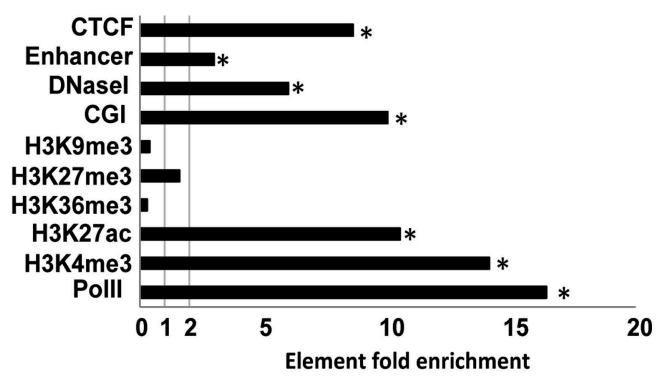

H
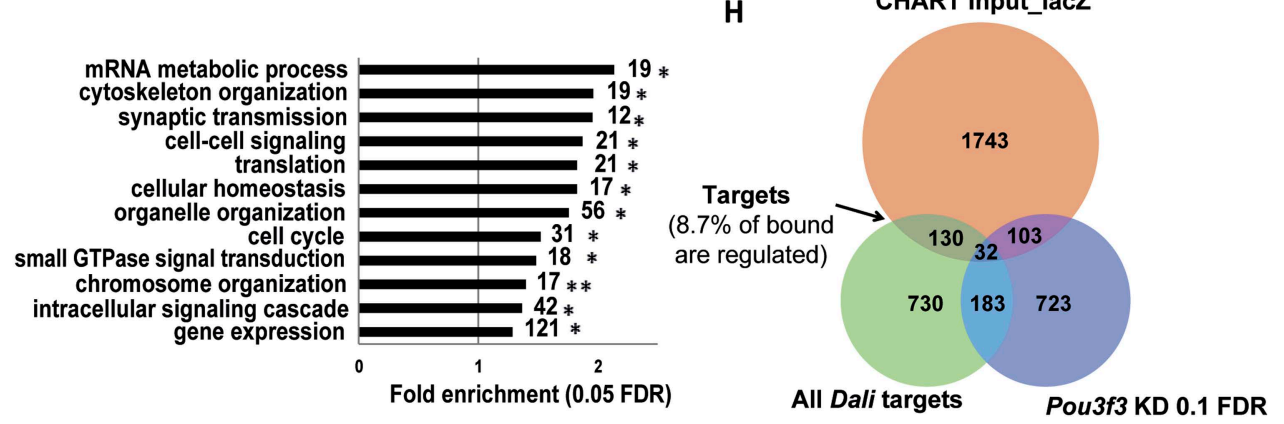

Figure 5. CHART-Seq analysis of Dali genomic binding sites. (A) Peaks were called against control CHART-seq experiments and input DNA. We consider only the 1427 peaks common to both comparisons (Supplementary File 5). (B) Sequencing of Dali bound DNA reveals focal peaks, including those at the promoter of Ache, E2f2, and Hmgb2. (C and D) Dali peaks are broadly distributed across the mouse genome (C) but are particularly enriched in 5' UTRs and gene promoters (D). Red arrowheads in (C) mark the Dali locus. (E) A third of Dali peaks Figure 5. Continued on next page 
Figure 5. Continued

are situated within $5 \mathrm{~kb}$ of a TSS. (F) Dali-bound loci are enriched in active chromatin marks (H3K4me3, H3K27ac, PoIII), DNase I hypersensitivity regions, enhancers and CpG islands annotations (CGI), and CTCF-bound regions, while being depleted of gene body marks (H3K36me3) and repressive chromatin marks (H3K9me3 and H3K27me3). (G) Representative categories from GO analysis of genes associated with Dali binding sites (within $1 \mathrm{Mb}$ ) include gene expression, cell cycle, signalling, synaptic transmission and cytoskeleton organization among others. Categories marked with an asterisk $\left(^{\star}\right)$ are significantly enriched also among genes associated with peaks within $10 \mathrm{~kb}$ of a TSS, with two asterisks $\left.{ }^{(*}\right)$-among genes with peaks within $100 \mathrm{~kb}$ (Supplementary File 5). (H) The intersection of genes proximal (<1 Mb) to Dali peaks, regulated by Dali and changing expression upon Pou3f3 (10\% FDR) knockdown identifies those both bound and regulated by Dali, as well as genes regulated by both Dali and Pou3f3 and directly bound by Dali.

DOI: 10.7554/eLife.04530.011

The following figure supplement is available for figure 5:

Figure supplement 1. CHART Analysis.

DOI: 10.7554/eLife.04530.012

however, suggests that it may be able to regulate DNMT1-mediated CpG methylation at CpG islandassociated promoters of Dali-bound and -regulated genes in trans. To investigate this, we performed Combined Bisulfite Restriction Analysis (COBRA) (Xiong and Laird, 1997) in parallel at 10 different $\mathrm{CpG}$ islands. Selection of these regions was on the basis that they each contained several COBRAcompatible restriction enzyme sites and could be efficiently amplified from bisulfite-converted template. COBRA demonstrated that five of these regions (corresponding to four genes) exhibited altered restriction profiles indicative of altered DNA methylation status after Dali depletion depletion (Figure 7-figure supplement 1). The inability of COBRA to detect changes at all sites may indicate that the DNA methylation status of the remaining regions did not change upon Dali depletion or that changes that occurred were undetected due to technical limitations of the assay.

Bisulfite sequencing demonstrating that the Dlgap5, Hmgb2, and Nos1 promoters each display increased CpG methylation in two independent stable Dali knockdown lines compared to control further confirmed these results (Figure 7A). Importantly, these data show that methylation changes occur within the core of these CpG islands and are not limited to their shores. Although other unidentified factors are also likely to play a role, our results are consistent with Dali (or a Dali:POU3F3 complex) acting in trans, as part of a multi-subunit ribonucleoprotein complex, to reduce DNMT1mediated $\mathrm{CpG}$ methylation at a subset of bound and regulated gene promoters away from its site of transcription.

One of these genes, Nos1, has multiple alternative promoters falling into two distinct regions (for simplicity referred to here as Exon 1 and Exon 2) whose differentiated use is proposed to fine-tune its expression in response to various physiological and developmental stimuli (Bros et al., 2006). Only the $5^{\prime}$-most region contains a CpG island and is bound by Dali (Figure 7B). By measuring expression levels of the three $5^{\prime}$-most Nos 1 exons in stable Dali knockdown and control lines we observed that the expression level of the $5^{\prime}$ most Dali-bound Exon 1 was reduced, relative to that for Exon 3, when Dali was depleted, whereas the expression ratio between Exons 2 and 3 was unaffected (Figure 7C). The preferential use of the $5^{\prime}$ most $\mathrm{CpG}$ site could reflect a secondary effect of Dali knockdown. Nevertheless, the observation that this site is bound by Dali transcript suggests that Dali may function by promoting the preferential use of a distantly located (and more rarely used) alternative promoter potentially through its effect on promoter-associated CpG island methylation.

\section{Dali and POU3F3 protein form a trans-acting transcriptional regulatory complex}

A recognisable binding motif for POU III family transcription factors, such as POU3F3, was present in 115 out of 1427 Dali CHART-Seq peaks ( $8.0 \%$; E-value $=3.8 \times 10^{-5}$; Figure 6F). This finding, together with Dali and Pou3f3 regulating a set of common genes (Figure 3D) and Dali occupying regulatory regions within 135 (13\%) of Pou3f3 targets (Figure $5 H$ ), suggested that Dali and POU3F3 protein may interact physically. Indeed, we observed direct RNA-protein interactions between over-expressed FLAG-tagged POU3F3 and co-transfected Dali, using UV-RIP in N2A cells (Figure 6G). Using ChIPqPCR, we then determined that at least five genes that were regulated by both Dali and Pou3f3 contained regions that were bound both by Dali and by POU3F3 protein (Figure $6 \mathrm{H}$ ). These results 
A

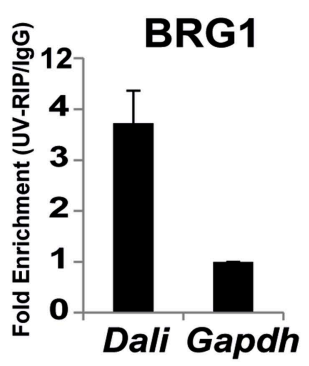

C

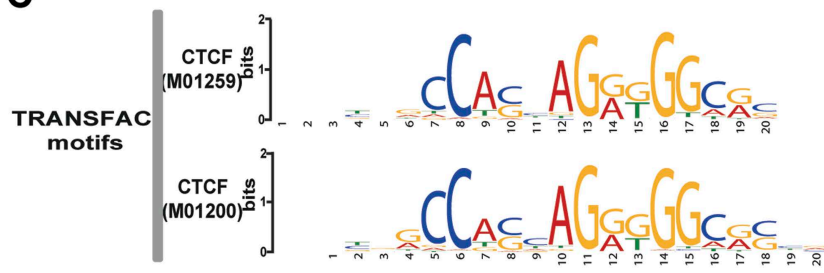

E

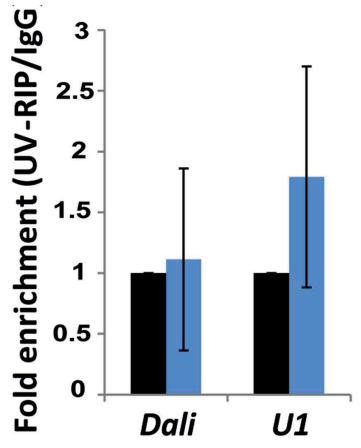

G
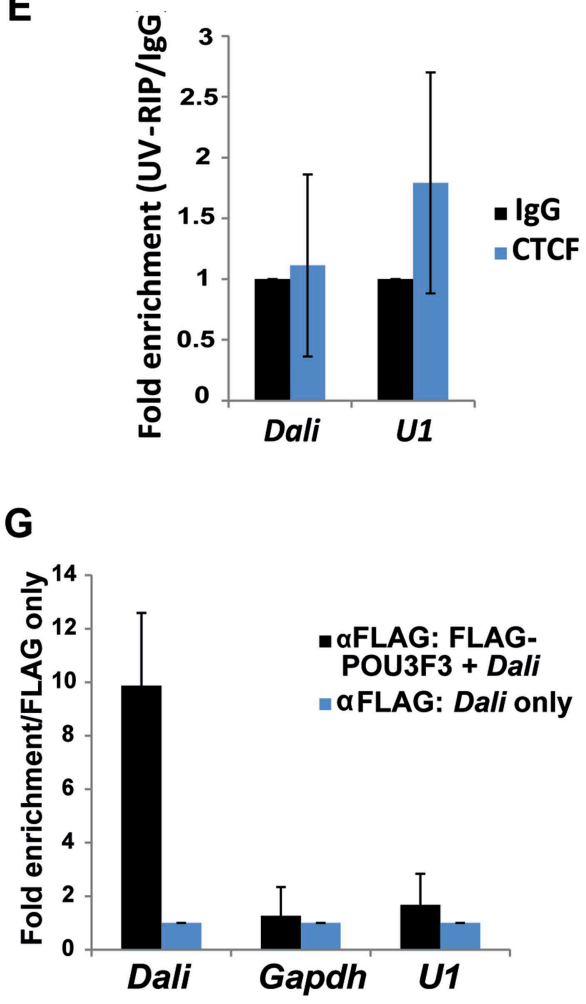

B

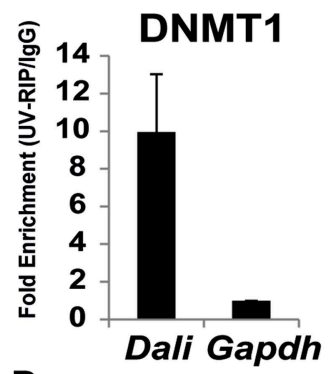

D
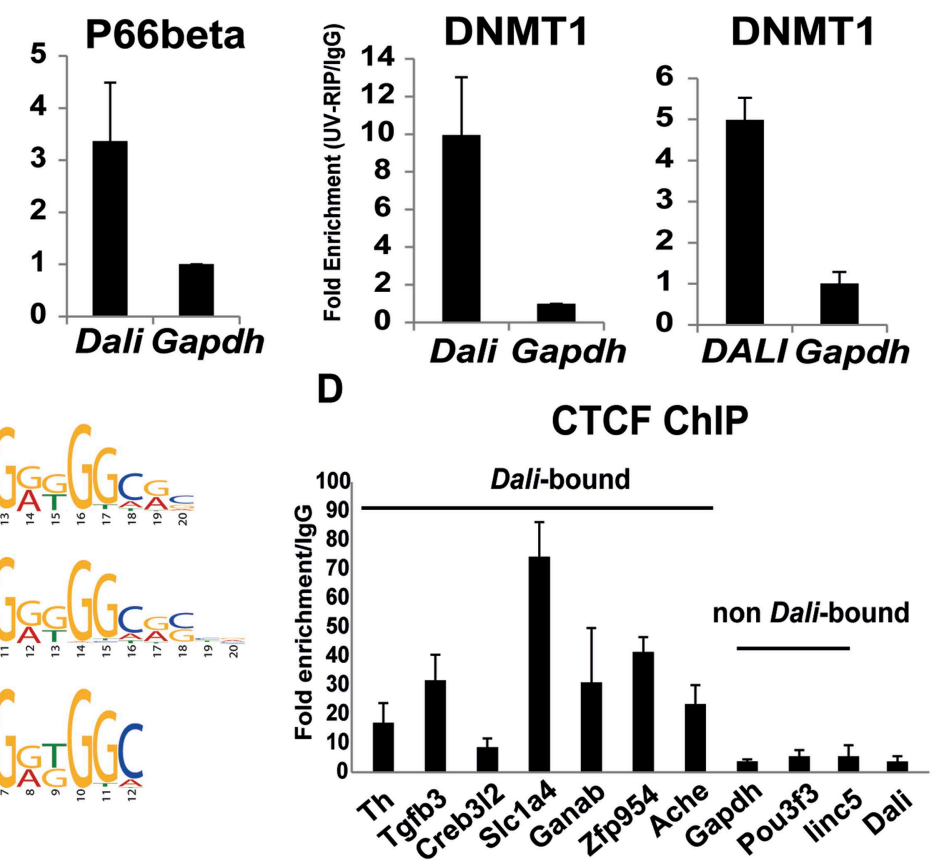

\section{CTCF ChIP}

F

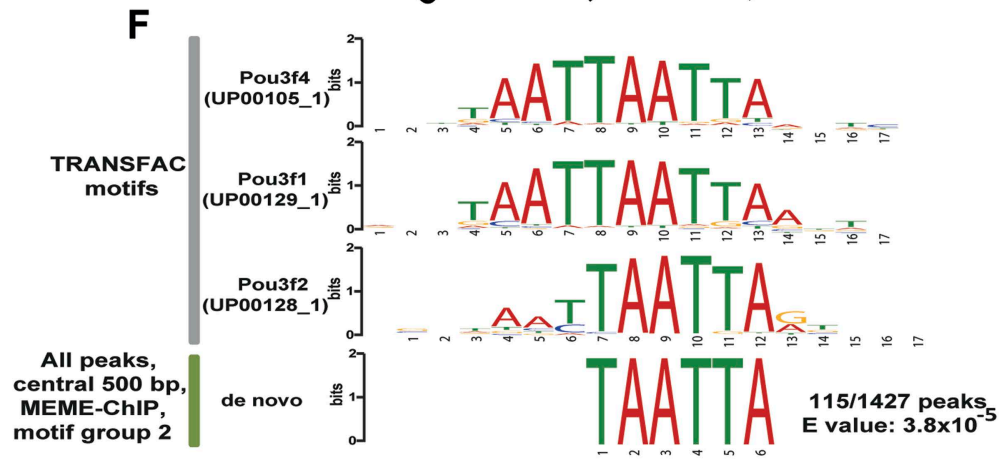

H

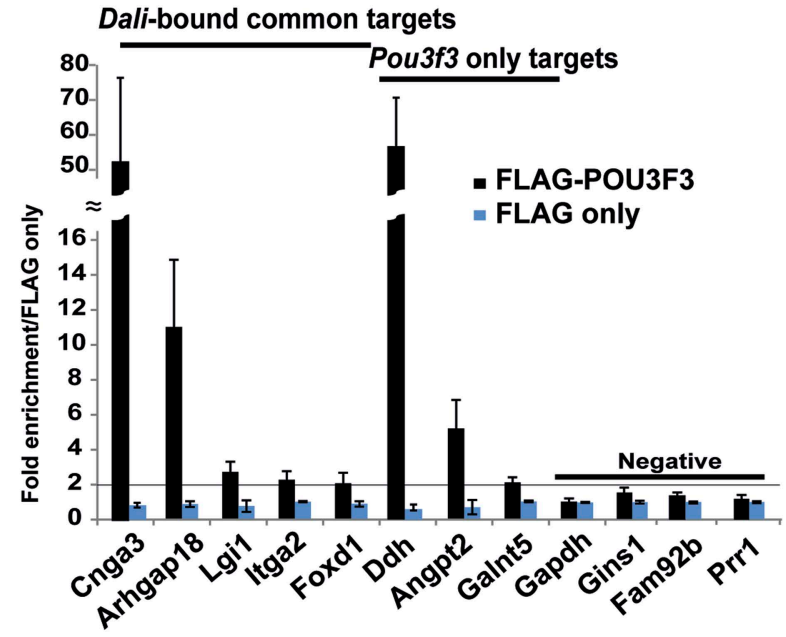

Figure 6. Dali associates with chromatin and transcriptional regulatory proteins. Dali interacts with BRG1, SIN3A, and P66beta in mouse N2A cells (A) and DNMT1 in mouse N2A and human SH-SY5Y cells (B). Nuclear extracts prepared from UV cross-linked cells were immuno-precipitated using either anti-DNMT1 or control lgG antibodies. Associated RNAs were purified and the levels of Dali and control Gapdh mRNA were quantified using Figure 6. Continued on next page 
Figure 6. Continued

qRT-PCR. Results are expressed as fold enrichment relative to an isotype lgG control antibody. Mean value \pm s.e., $n=3$. (C) De novo discovery of a near-perfect match to a CTCF motif in 125/1427 (8.8\%) Dali CHART-Seq peaks. (D) Dali co-occupies several locations shared with CTCF. Control regions are not predicted to be bound by CTCF and are not bound by Dali. ChIP assays were performed in N2A cells using either an antibody against CTCF or an isotype specific control. The indicated DNA fragments were amplified using qPCR. Fold enrichment was calculated as 2- $\Delta \Delta C \mathrm{Ct}$ (IP/lgG). Mean value \pm s.e., $n=3$. (E) Dali does not directly interact with CTCF protein in mouse N2A cells. Nuclear extracts were prepared from UV cross-linked cells and immuno-precipitated using either anti-CTCF or control IgG antibodies. Associated RNAs were purified and the levels of Dali and control U1 snoRNA were detected in each UV-RIP using qRT-PCR. Results are expressed as fold enrichment relative to an isotype lgG control antibody. Results are presented as mean value \pm s.e. of three independent experiments. (F) De novo discovery of a motif for POU III family transcription factors (which includes POU3F3) in 115/1427 (8.1\%) Dali CHART-Seq peaks. (G) UV-RIP in N2A cells: FLAG-tagged POU3F3 protein directly interacts with Dali. Mean value \pm s.e., $\mathrm{n}=3$. (H) ChIP-qPCR in N2A cells: POU3F3 occupies a subset of loci bound by Dali and regulated by both Pou3f3 and Dali. Loci associated with known (Dali-independent) Pou3f3 targets were used as positive control, while loci not regulated by either Pou3f3 or Dali and not bound by Dali were used as negative control. Mean value \pm s.e., $\mathrm{n}=3$.

provide further mechanistic insights into Dali's mode of action and indicate that Dali and POU3F3 form a complex that binds to and regulates a subset of genes in trans in N2A cells.

\section{Induction of the endogenous Dali transcript in mouse ES cells regulates Pou3f3 locally and E2f2 distally}

Finally, we tested whether de novo expressed Dali transcript can act as a transcriptional regulator in order to further substantiate the observation that Dali functions as a novel regulator of both local and distal gene expression. To achieve this, we induced Dali expression from its endogenous locus in E14 mouse ES cells, which do not express Dali or Pou3f3 to detectable levels, using transient transfection of an artificial Transcription Activator-Like effector (TALE) transcription factor. After $72 \mathrm{hr}$, up-regulation of Dali transcript was shown to significantly increase Pou3f3 expression (Figure 7D). Dali expression from its own locus is thus sufficient to induce the expression of its genomically neighbouring Pou3f3 gene (Figure 7D). We next investigated the expression levels of E2f2, a gene that we found to be negatively regulated by Dali using shRNA mediated knockdown (Supplementary file 2), and found that Dali up-regulation reduced E2f2 transcript levels by approximately 40\% (Figure 7D). Taken together, these results indicate that Dali can regulate both local and distal target genes when its expression is induced from its endogenous locus.

\section{Discussion}

The ability of nuclear localised IncRNAs to act in trans at distal genomic locations to regulate gene expression programs has been poorly understood. This is in large part because the direct transcriptional targets of only a small number of such transcripts (for example, Paupar (mouse), HOTAIR, NEAT1, TERC, RMST (all human), and rox2 (Drosophila)) have been identified thus far (Chu et al., 2011; Simon et al., 2011; Ng et al., 2013; Vance et al., 2014). Consequently, it has been unclear how these transcripts are targeted to distal functional elements and whether thereafter they alter chromatin structure in situ.

In this study we found evidence that the intergenic IncRNA Dali acts both locally to regulate the expression of its nearest protein-coding gene, Pou3f3, and distally to regulate both Pou3f3-dependent and -independent target genes in an RNA-dependent manner. 8.8\% (150) of all genes whose expression altered following Dali depletion were associated with Dali binding sites within $1 \mathrm{Mb}$ (although $30 \%$ of peaks reside within $5 \mathrm{~kb}$ of a TSS, see Figure 5E) and, therefore, are likely to represent direct regulatory targets. This proportion lies within the range of functional sites observed for transcription factors (Cusanovich et al., 2014). Our results are consistent with a model in which mouse or human Dali is recruited to chromatin indirectly via RNA-protein interactions with both sequence-specific transcription factor proteins, such as POU3F3 which is encoded by its neighbouring gene, or non-sequence specific DNA binding cofactors including DNMT1, which in turn may interact with sequence-specific DNA-binding proteins. In this model, Pou3f3-dependent target genes are regulated by Dali both indirectly, via its transcriptional regulatory effect on the Pou3f3 gene, and directly via its physical interaction with the POU3F3 protein and their co-occupancy at regulatory regions of target genes.

Our data show that both human and mouse Dali associate with DNMT1 and that depletion of Dali levels increases CpG methylation at Dali bound and regulated promoters in trans. Whilst a growing 
A
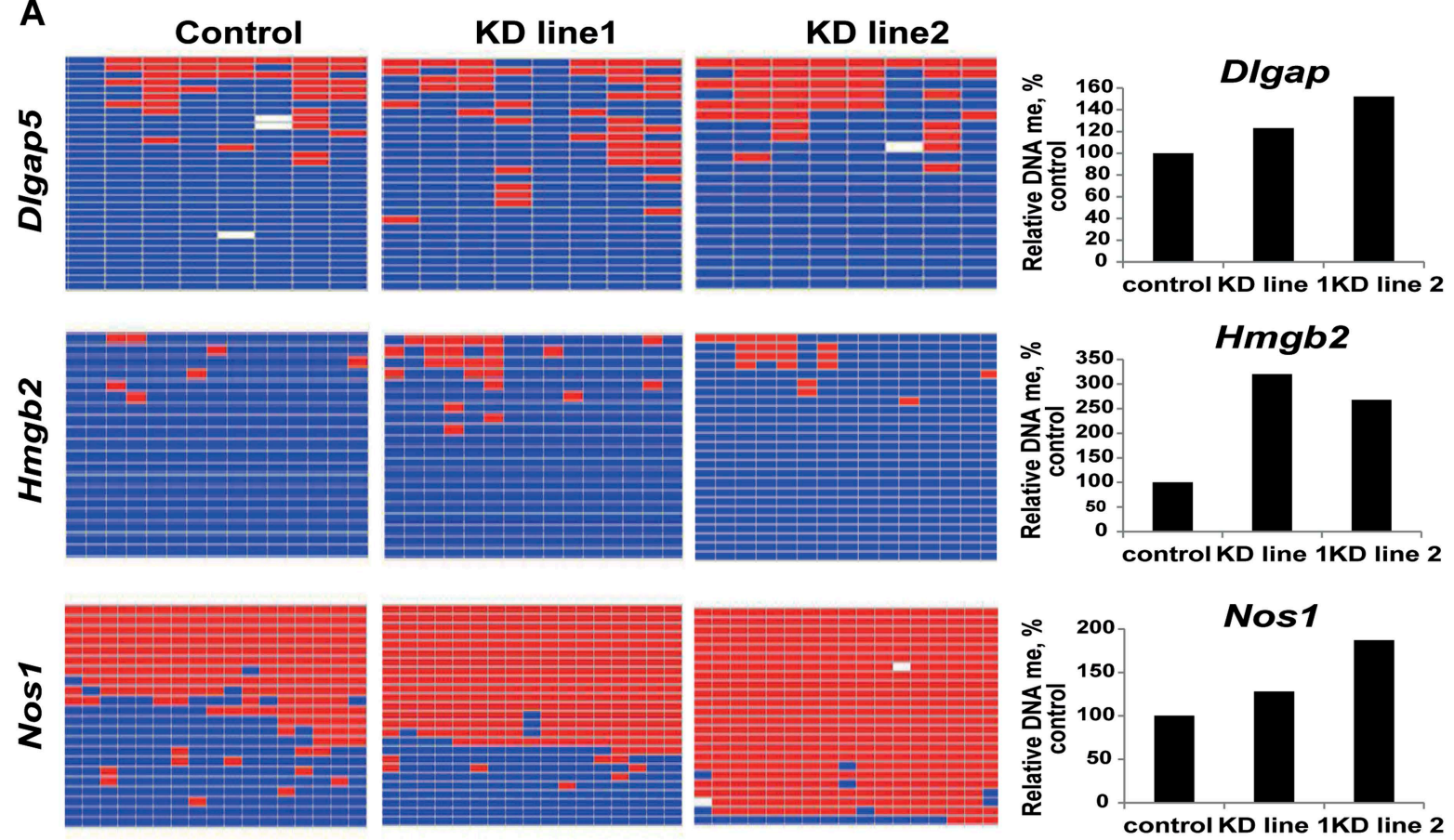

B

$\operatorname{chr} 5(\mathrm{~mm} 9)$

$118,295,000$

$118,300,000$ ।

$118,305,000$

$118,310,000$ ।

$118,315,000$

Exon 1

CpG ${ }^{\text {Nos1 }}$

C

\section{Dali knockdown (stable)}

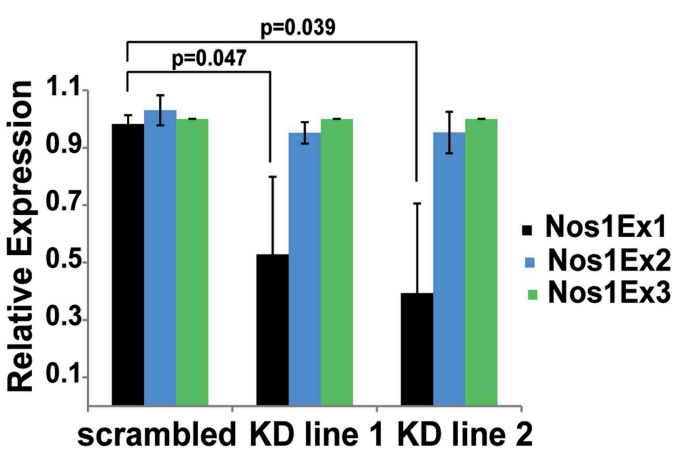

D

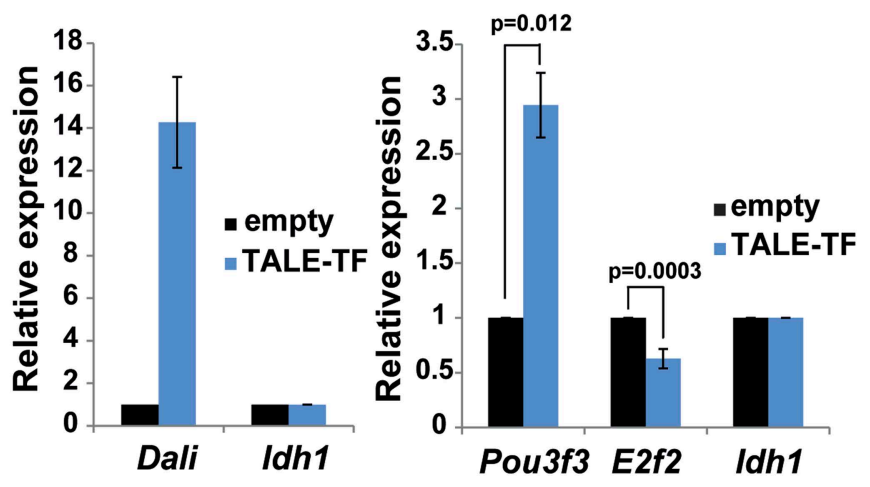

Figure 7. Dali modulates DNA methylation at bound and regulated promoters. (A) DNA methylation status of three CGI-associated promoters bound and regulated by Dali was assessed using bisulfite sequencing in control and two stable Dali knockdown lines. DNA methylation levels were found to be increased in knockdown lines. The degree of increase was correlated with the degree of Dali knockdown (see Figure 7-figure supplement 1).

(B) Nos1 gene has two clusters of alternative TSSs (Exon 1 and Exon 2). The upstream neuronal tissue-specific cluster (Exon 1) is associated with a CpG island and is bound by Dali. (C) Down-regulation of Nos1 observed in stable Dali knockdown lines can be explained by reduced initiation from the Dali-bound TSS (Exon 1), as the ratio between Exon1 and an internal Exon 3 is diminished, while the ratio between Exon 2 and Exon 3 is not changed. Mean values \pm s.e, $n=3$, one tailed t-Test, unequal variance. (D) Dali transcript regulates Pou3f3 locally and E2f2 distally in ES mouse cells. Dali is expressed from its endogenous locus in non-expressing mouse E14 ES cells using custom-designed TALE-TF (left). De novo induction of the endogeFigure 7. Continued on next page 
Figure 7. Continued

nous Dali locus is sufficient to up-regulate the neighbouring Pou3f3 gene and down-regulate the distally located E2f2 gene (right). Mean

value \pm s.e., $\mathrm{n}=3$.

DOI: 10.7554/eLife.04530.014

The following figure supplement is available for figure 7:

Figure supplement 1. DNA Methylation analysis.

DOI: 10.7554/eLife.04530.015

body of literature has implicated IncRNAs, such as Kcnq1ot1 and ecCEBPA (Mohammad et al., 2010; Di Ruscio et al., 2013), in modulating CpG methylation in a DNMT1-dependent manner at their sites of synthesis, our findings represent the first evidence that an intergenic IncRNA can regulate DNA methylation in trans at distal genomic locations away from its site of transcription.

Our findings suggest that Dali inhibits DNA methylation at a subset of bound and regulated regions, presumably deposited by the DNMT1 DNA methyltransferase, to which it binds. DNMT1 binds structured RNA with higher affinity than its DNA substrate (Di Ruscio et al., 2013). It is thus possible that Dali competes for binding to DNMT1 with either protein co-factors such as UHRF1, which loads and orients the enzyme on the DNA substrate (Inomata et al., 2008), or its DNA substrate. Targeting of DNMT1 to specific loci is believed to be mediated by DNMT1-interacting transcription factors. 58 transcriptional factors have been reported as DNMT1 interactors (Hervouet et al., 2010), of which 9 have enriched sequence motifs in Dali CHART-Seq peaks. We thus propose a model in which such transcription factors promote the sequence-specificity of Dali-modulated DNA methylation changes. The genomic co-localisation of DNMT1 and transcription factors using ChIP remains unknown owing to the poor performance of the available anti-DNMT1 antibodies in this application.

We have shown that Dali regulates genes involved in neural development and function and its depletion disrupts terminal stages of neuronal differentiation, more particularly neurite outgrowth development. Dali RNA binds to and up-regulates the promoters or promoter-proximal regions of key pro-differentiation factors, such as E2f2 (Persengiev et al., 2001), Fam5b (Terashima et al., 2010), Sparc (Bhoopathi et al., 2011) and Dkk1 (Cajanek et al., 2009) (Watanabe et al., 2005), as well as binding and negatively regulating genes such as Kif2c and Kif11 which are known to block neurite outgrowth (Laketa et al., 2007; Myers and Baas, 2007; Nadar et al., 2012). Therefore, Dali works as a pro-differentiation factor in neural development by regulating the balance between proliferation and differentiation, as well as processes associated with terminal neuronal differentiation.

Cis- or trans-acting modes of action have been proposed for a growing number of IncRNAs (Fatica and Bozzoni, 2014). Dali is unusual in acting in a transcript-dependent manner to perform both local and distal gene regulatory roles like another such IncRNA, Paupar (Vance et al., 2014). Dali is transcribed in the vicinity of a neuronal transcription factor Pou3f3. Both Dali and Paupar IncRNAs are CNS-expressed and evolutionarily constrained transcripts that are co-expressed with their neighbouring transcription factor genes both spatially and temporally. Moreover, both IncRNAs interact directly with the protein product of their neighbouring genes, POU3F3 and PAX6, respectively, to regulate a large set of targets in trans. These observations, together with the preferential genomic location of intergenic IncRNA loci adjacent to transcription factor genes (Ponjavic et al., 2009) imply that IncRNAs may commonly interact with the product of genomically adjacent transcription factor genes to act in trans on distal genes.

\section{Materials and methods}

\section{Plasmid construction}

We used the Whitehead Institute siRNA selection program to design shRNAs that target multiple regions of Dali or Pou3f3. To minimise the possibility of off-target effects, we compared candidate sequences against the $\mathrm{NCBI}$ RefSeq database and removed those with $\geq 15$ bases in the anti-sense strand that matched a database entry. We then cloned the double stranded DNA oligonucleotides containing sense-loop-antisense targeting sequences downstream of the U6 promoter in pBSU6-CMVeGFP (Sarker et al., 2005) by linker ligation. The Dali expression plasmid was constructed by PCR amplifying the full length Dali sequence as an EcoRI-Xhol fragment from mouse N2A cell genomic DNA and inserting it into pcDNA3. The FLAG-tagged Pou3f3 expression plasmid was 
constructed by excising the full length Pou3f3 ORF from Pou3f3 (NM_008900) mouse cDNA clone in pCMV Entry vector (Cambridge Biosciences, UK) and inserting it into the multiple cloning site (MCS) of the N-terminal pFLAG-CMV-6a vector (Sigma-Aldrich, UK) between EcoRI and EcoRV sites. The sequences of all oligonucleotides used for cloning are shown in Supplementary file 1.

\section{Dali and Pou3f3 knockdown}

Cells were plated at a density of approximately $2 \times 10^{5}$ cells per well in a six well plate. $16-24 \mathrm{hr}$ later cells were transfected with $1.5 \mu \mathrm{g}$ shRNA expression construct using FuGENE 6 (Promega, UK) following the manufacturer's instructions. Total RNA was extracted from the cells $48-72 \mathrm{hr}$ later using TRIzol-chloroform extraction method. For stable transfections, N2A cells were co-transfected with the pBSU6-shRNA expression vector and pTK-Hyg (Clontech, Mountain View, CA) at a 5:1 ratio. $72 \mathrm{hr}$ post-transfection $200 \mu \mathrm{g} / \mathrm{ml} \mathrm{Hygromycin} \mathrm{B}$ was added to the cells to select individual drug resistant clones that were later isolated and expanded under selective conditions. Dali expression in individual clones was measured by qRT-PCR.

\section{qRT-PCR and RACE}

Reverse transcription was performed using the QuantiTect Reverse Transcription Kit (Qiagen, Netherlands). SYBR Green quantitative PCR was performed using a Step One Plus Real-Time PCR System (Applied Biosystems, UK). For RACE, GeneRacer Kit (Invitrogen, UK) was used according to the manufacturer's instructions. Human foetal brain RNA was purchased from Promega. Primers are listed in Supplementary file 1.

\section{Cell culture}

Mouse N2A neuroblastoma and E14 ES cells were cultured as described in (Vance et al., 2014). The N2A cell line was chosen because it has been used extensively as a model to study neural differentiation in vitro (Shea et al., 1985). Human neuroblastoma (SH-SY5Y) cells were grown in DMEM/F12 medium supplemented with $10 \% \mathrm{FBS}, 1 \%$ penicillin-streptomycin, and $1 \% \mathrm{~L}$-glutamine at $37^{\circ} \mathrm{C}$ in a humidified atmosphere with $5 \% \mathrm{CO}_{2}$. Biochemical fractionation, ChIP and UV-RIP experiments was performed exactly as described in Vance et al. (2014). The following antibodies were used: antiDNMT1 (ab87656; Abcam, UK), anti-BRG1 (ab4081; Abcam), anti-P66beta (ab76924; Abcam), antiSIN3A (Active Motif, Belgium, 39,865), anti-CTCF (Abcam, 70,303), anti-rabbit lgG control antibodies (Millipore, Billerica, MA) and mouse monoclonal anti-FLAG M2 beads (Sigma-Aldrich) for FLAGtagged POU3F3 experiments.

\section{Animal work}

All animal experiments were conducted in accordance to schedule one UK Home Office guidelines (Scientific Procedures Act, 1986). C57BL/6J, postnatal day P56 male and pregnant females were killed by cervical dislocation; whole brains were dissected in ice-cold phosphate-buffered saline (PBS) from adult $(n=2)$, and intrauterine stages E9 $(n=6)$, E10.5 $(n=6)$, E13.5 $(n=6)$, E15.5 $(n=6)$ and E18.5 $(n=6)$ mice. Brains were embedded in $5 \%$ agarose (low melting, Bioline) and sectioned using a vibrating microtome (Leica, VT1000S) into $200 \mu \mathrm{m}$ coronal sections using a chilled solution of 1:1 mixture of RNAlater (Ambion) and PBS. Regions of interest (adult: dentate gyrus, subventricular zone and olfactory bulb; embryos: preplate, proliferative compartmenst combining ventricular and subventricular zones, and cortical plate from lateral and dorsal tiers) were dissected from individual sections using 27 gauge needles under visual guidance, using transillumination on a dissecting microscope (MZFLIII, Leica, Switzerland). Dissected samples were rinsed in RNAse free PBS/RNAlater 1:1, submerged in ice-cold RNAlater kept for $24 \mathrm{hr}$ at $4^{\circ} \mathrm{C}$ and stored at $-80^{\circ} \mathrm{C}$ in RNAlater until processing.

\section{Transcriptomic analysis}

Total RNA was isolated using the Qiagen Mini RNeasy kit according to the manufacturers' instructions. RNA integrity was assessed on a BioAnalyzer (Agilent Technologies, UK). 200 ng RNA was used to produce labelled sense single stranded DNA (ssDNA) for hybridization with the Ambion WT Expression Kit, the Affymetrix WT Terminal Labelling and Controls Kit and the Affymetrix Hybridization, Wash, and Stain Kit following the manufacturer's instructions. Sense ssDNA was fragmented and the distribution of fragment lengths was assessed on a BioAnalyzer. Next, fragmented ssDNA was labelled and hybridized to the Affymetrix GeneChip Mouse Gene 1.0 ST Array (Affymetrix, UK). Arrays were processed on an Affymetrix GeneChip Fluidics Station 450 and Scanner 3000. 
CEL files were analysed using the Limma, oligo, and genefilter R Bioconductor packages (Smyth, 2004; Carvalho and Irizarry, 2010). Arrays were RMA background corrected and quantile normalised. Summary expression values were calculated the gene level. Genes whose expression changed upon Dali and Pou3f3 knockdown, as well as upon retinoic acid induced differentiation of control and stable Dali knockdown cells, were filtered to remove genes showing little variation in expression (variance cut off of 0.5 ) before the identification of significant changes. In every case, the Limma Ebayes algorithm was used to identify differential expression between three knockdown and three control samples (biological replicates). 1.3-fold change cutoff was applied in every case. GOToolbox was used to perform Gene Ontology analyses ((Martin et al., 2004); http://genome.crg.es/GOToolBox/). Representative significantly enriched categories were selected from a hypergeometric test with a Benjamini-Hochberg corrected p-value threshold of 0.05 .

\section{CHART}

CHART Enrichment and RNase H Mapping experiments were performed as described in (Simon, 2013). We designed 10 biotinylated DNA capture (C)-oligos: 5 oligos complementary to the most accessible regions of Dali, as determined by RNase $\mathrm{H}$ mapping, and 5 oligos targeting the most evolutionarily conserved regions of the transcript (Figure 5A). These oligos were used as two cocktails of 5 oligos, and as a pool of all 10. As controls, we used an oligo designed to target the antisense Dali sequence (absent from the N2A transcriptome). Additionally we require peaks to not overlap with those identified in an analogous CHART-sequence experiment using the $E$. coli lac $Z$ sequence (GSE52571) (Vance et al., 2014). Compared to controls, all three cocktails of Dali oligos showed significant enrichment of the Dali transcript (10-fold compared to lacZ), but no enrichment of the abundant mRNA Gapdh (Figure 5B). Without any prior information about Dali genomic binding, we considered its endogenous site of synthesis to assess the enrichment of transcript-associated DNA loci. Specific enrichment of Dali at its locus was observed as expected (Figure 5-figure supplement 1).

CHART extract was prepared from approximately $3 \times 10^{8} \mathrm{~N} 2 \mathrm{~A}$ cells per pull down and hybridized overnight with 810 pmol biotinylated oligonucleotide cocktail (Supplementary File 1) at room temperature with rotation. $250 \mu \mathrm{l} \mathrm{MyOneC1}$ streptavidin beads (Invitrogen) were used to capture the complexes overnight at room temperature with rotation. After extensive washes, bound material was eluted using RNase H (New England Biolabs (NEB), UK) for $30 \mathrm{~min}$ at room temperature. Samples were treated with Proteinase $K$ and cross-links were reversed. RNA was purified from 1/5 total sample volume using the QIAGEN miRNeasy kit. DNA was prepared from the remaining sample using the phenol:chloroform:isoamyl alcohol extraction and ethanol precipitation method. DNA was further sheared to an average fragment size of 150-300 bp using a Bioruptor (Diagenode, Belgium) and sequenced on an Illumina HiSeq (50 bp paired end).

\section{Computational analysis of CHART-seq data}

CHART-seq was performed with three independent pull down samples (using two independent cocktails of $5 \mathrm{C}$-oligos, and one cocktail containing all $10 \mathrm{C}$-oligos) and sequenced simultaneously with a matched input sample. $50 \mathrm{bp}$, paired-end reads were mapped to the mouse genome (mm9) using bowtie with the options '-m1 -v2 -best-strata-a'. For each Dali sample, peaks were called against the matched N2A input sample (4208 peaks) and CHART-seq peaks previously analogously identified in N2A cells using two lacZ controls (1928 peaks) (Vance et al., 2014). Peak calls were made using the MACS2 algorithm ((Zhang et al., 2008); https://github.com/taoliu/MACS/blob/master/README) with the options '-mfold 1030 -gsize $=2.39 \mathrm{e} 9$-qvalue $=0.01$ ' using the CGAT pipeline 'pipeline_mapping.py' (https://github.com/CGATOxford/cgat). Peak calls were then filtered such that only peak calls with a $-\log 10 q$ value $>5$ were retained (FDR $0.001 \%$ ).

We discovered 1427 Dali-associated regions genome-wide called against both input and lacZ control samples (Figure 5A; Supplementary file 5).

\section{Characterisation of Dali binding sites}

The chromosomal distribution of Dali peaks was visualised using the R Bioconductor package 'ggbio' (Yin et al., 2012). Genome territory enrichments analysis was performed using the Genome Association Tester (GAT; (Heger et al., 2013)). 10,000 simulations were performed using a mappability filtered workspace and an isochore file partitioning the genome into eight bins based on regional GC content. For the chromosomal enrichment analyses, chromosomal territories were proportionally assigned to a 
single virtual meta-chromosome before using GAT to test for GC and mappability corrected enrichments as above. Gene Ontology categories enriched for Dali binding were identified by intersecting regulatory regions for known coding genes with Dali binding sites. Regulatory regions for genes were defined following the GREAT definition (McLean et al., 2010) as a basal domain surrounding the TSS (from $-5 \mathrm{~kb}$ to $+1 \mathrm{~kb}$ ) and extending domains upstream and downstream to the nearest gene's basal domain or to a maximum distance of $1 \mathrm{Mb}$. Enrichments were identified using GOToolbox.

Dali peaks were characterised using DNase I hypersensitivity (HS) data generated by the Stamatoyannopoulos lab at the University of Washington and chromatin features identified by the Ren lab at the Ludwig Institute for Cancer Research ((Shen et al., 2012); ENCODE Project Consortium, 2012). Enrichments of DNase I HS and chromatin features overlapping Dali peaks were assessed using GAT to control for mappability and regional GC content as above.

Complementarity between Dali sequence and binding locations was assessed using the EMBOSS Water algorithm (Rice et al., 2000) which performs Smith-Waterman alignment with a range of gap opening and extension penalties. RNA-DNA:DNA triplex formation was assessed using the Triplexator search software suit (Buske et al., 2012). The MEME-ChIP (Machanick and Bailey, 2011) algorithm was used to perform de novo motif discovery analysis by examining the unmasked DNA sequence of the central regions of peak locations. MEME-ChIP was run with the options '-mememod zoops -meme-minw 5 -meme-maxw 30-meme-nmotifs 50' using a custom background file prepared from regions flanking the peak locations using the command 'fasta-get-markov -m 2'. Enrichment of known vertebrate transcription factor binding sites from the TRANSFAC Professional database (Matys et al., 2006) was assessed using the AME algorithm (McLeay and Bailey, 2010) with the options '-method fisher-length-correct' using the sequence and background file prepared for MEME-ChIP analysis.

\section{$3 C$}

E14 ES cells or day 4 ES-derived neuronal were cross-linked with $2 \%$ formaldehyde. Nuclei were prepared and permeabilized with $0.3 \%$ SDS in $1.2 \times$ restriction buffer (NEB3 for BgllI) for $1 \mathrm{hr}$ at $37^{\circ} \mathrm{C}$. Then, SDS was sequestered by adding 1.8\% Triton X-100. $1 \times 10^{6}$ nuclei ( 15 $\mu \mathrm{g}$ of chromatin) were digested with 400 units of Bglll restriction enzyme overnight, and the enzyme was inactivated. Nuclei were diluted in 1.15× T4 DNA ligation buffer (NEB), and SDS sequestered by adding $1 \%$ Triton X-100. The digested chromatin was ligated using 100 Weiss units of T4 DNA ligase for $4 \mathrm{hr}$ at $16^{\circ} \mathrm{C}$ and treated with Proteinase $K$ to reverse cross-links. Samples were further treated with RNase A, and DNA was phenol-chloroform extracted and ethanol precipitated.

A RP23-92N4 (CHORI; BACPAC) Bacterial Artificial Chromosome (BAC) clone covering the Pou3f3-Dali locus was treated as above and used as a control template for the $3 \mathrm{C}$ assay. Ligation products of $3 \mathrm{C}$ and BAC samples were quantified by qPCR. PCR reactions consisted of $300 \mathrm{ng}$ $3 \mathrm{C}$ sample, $0.2 \mu \mathrm{M}$ test primers and a primer corresponding to Dali promoter and $1 \times$ SYBR Green PCR Mastermix (Life Technologies, UK). All reactions were performed in triplicate. The mean threshold cycle $(\mathrm{Ct})$ value was calculated and used to calculate relative amounts of PCR products. To normalise for different primer efficiencies, interaction frequencies were calculated by dividing the amount of PCR product obtained from the $3 C$ sample by the amount of DNA obtained from control BAC DNA. Interaction frequencies were also normalised to Gapdh internal controls prepared from genomic DNA in the same manner as the BAC clone sample. All primers used are listed in Supplementary file 1.

\section{COBRA}

We used COBRA to study 9 out of 44 CpG island-containing promoters bound by Dali and associated with genes differentially expressed between stable Dali knockdown and control cell lines prior to or subsequent to the RA-induced differentiation. 80-350 ng of genomic DNA was bisulfite-treated using EZ DNA Methylation Gold kit according to the manufacturer's instruction and used for PCR amplification. Primers for amplifying bisulfite converted template DNA were designed using MethPrimer software accessible at http://www.urogene.org/methprimer/ (Li and Dahiya, 2002). PCR products were on-column purified with QIAquick PCR Purification Kit. $250 \mathrm{ng}$ to $1 \mathrm{\mu g}$ of purified products were incubated with appropriate COBRA-compatible (BstUI (NEB), Mspl (NEB), Taql (Thermo Scientific), HpyCH4IV (NEB)) or control (Hsp92II (Promega), Bfal (NEB)) restriction enzymes overnight. Restriction products were analysed on $3 \%$ low melting point agarose gels. 


\section{TALE-mediated up-regulation}

Target regions were selected and TAL effector constructs were designed using software, tools, and information found on the TAL Effector Nucleotide Targeter 2.0 website accessible from https://tale-nt.cac.cornell.edu/. Construction of custom TALE-TFs designed to target promoterproximal region of Dali to up-regulate transcription from the locus was performed as described by Sanjana et al. (2012). The TALE-TF was designed to target the following region lying upstream of the TSS of Dali: chr1 (mm9): $42807019-42807038$ ("TGTCCCTTGTCCACATATCT"). The TAL domain sequence used was as follows: NH NG HD HD HD NG NG NH NG HD HD NI HD NI NG NI NG.

\section{Data deposition}

Microarray and CHART-Seq data have been deposited in the GEO database under accession number GSE62035 (http://www.ncbi.nlm.nih.gov/geo/query/acc.cgi?acc=GSE62035).

\section{Acknowledgements}

We thank the High-Throughput Genomics Group at the Wellcome Trust Centre for Human Genetics for the generation of the sequencing data and OXION for use of their microarray facility. This project has been funded by the European Research Council (Project Reference 249869, DARCGENs; KWV, VC, LK), the Medical Research Council (CPP, SNS; and MRC Hub Grant G0900747 91070 for Sequencing) and the Wellcome Trust (Grant Reference 090532/Z/09/Z for Sequencing). VC is a recipient of The Darwin Trust of Edinburgh Postgraduate research Scholarship.

\section{Additional information}

Competing interests

CPP: Senior editor, eLife. The other authors declare that no competing interests exist.

Funding

\begin{tabular}{|c|c|c|}
\hline Funder & Grant reference number & Author \\
\hline European Research Council (ERC) & 249869, DARCGENs & $\begin{array}{l}\text { Vladislava Chalei, } \\
\text { Lesheng Kong, } \\
\text { Keith W Vance }\end{array}$ \\
\hline Medical Research Council (MRC) & & $\begin{array}{l}\text { Stephen N Sansom, } \\
\text { Chris P Ponting }\end{array}$ \\
\hline The Darwin Trust of Edinburgh & Postgraduate Scholarship & Vladislava Chalei \\
\hline European Commission (EC) & Human Brain Project & Juan F Montiel \\
\hline
\end{tabular}

The funders had no role in study design, data collection and interpretation, or the decision to submit the work for publication.

Author contributions

VC, Conception and design, Acquisition of data, Analysis and interpretation of data, Drafting or revising the article; SNS, Analysis and interpretation of data, Drafting or revising the article; LK, Analysis and interpretation of data, Contributed unpublished essential data or reagents; SL, Acquisition of data, Contributed unpublished essential data or reagents; JFM, Acquisition of data, Analysis and interpretation of data; KWV, Conception and design, Analysis and interpretation of data, Drafting or revising the article, Contributed unpublished essential data or reagents; CPP, Conception and design, Drafting or revising the article

Author ORCIDs

Juan F Montiel, (iD http://orcid.org/0000-0001-8919-4662

Chris P Ponting, (iD http://orcid.org/0000-0003-0202-7816

Ethics

Animal experimentation: All animal experiments were conducted in accordance to schedule one UK Home Office guidelines (Scientific Procedures Act, 1986). 


\section{Additional files}

Supplementary files

- Supplementary file 1. Oligonuleotides.

DOI: 10.7554/eLife.04530.016

- Supplementary file 2. Transient Dali knockdown microarray profiling.

DOI: 10.7554/eLife.04530.017

- Supplementary file 3. Genes changing in transient Pou3f3 knockdown and intersection with Dali targets.

DOI: 10.7554/eLife.04530.018

- Supplementary file 4. Stable Dali knockdown gene lists.

DOI: 10.7554/eLife.04530.019

- Supplementary file 5. CHART analysis.

DOI: 10.7554/eLife.04530.020

- Supplementary file 6. Motif discovery.

DOI: 10.7554/eLife.04530.021

- Supplementary File 7. Mass spectrometry identification of Dali associated proteins.

DOI: 10.7554/eLife.04530.022

Major dataset

The following dataset was generated:

\begin{tabular}{|c|c|c|c|c|}
\hline Author(s) & Year & Dataset title & $\begin{array}{l}\text { Dataset ID } \\
\text { and/or URL }\end{array}$ & $\begin{array}{l}\text { Database, license, } \\
\text { and accessibility } \\
\text { information }\end{array}$ \\
\hline $\begin{array}{l}\text { Chalei V, Sansom SN, Kong L, } \\
\text { Lee S, Montiel J, Vance KW, } \\
\text { Ponting CP }\end{array}$ & 2014 & $\begin{array}{l}\text { Data from: The DNMT1 } \\
\text { associated IncRNA Dali is } \\
\text { an epigenetic regulator of } \\
\text { neural differentiation }\end{array}$ & GSE62035 & $\begin{array}{l}\text { Publicly available at } \\
\text { NCBI Gene Expression } \\
\text { Omnibus. }\end{array}$ \\
\hline
\end{tabular}

The following previously published dataset was used:

\begin{tabular}{|c|c|c|c|c|}
\hline Author(s) & Year & Dataset title & $\begin{array}{l}\text { Dataset ID } \\
\text { and/or URL }\end{array}$ & $\begin{array}{l}\text { Database, license, } \\
\text { and accessibility } \\
\text { information }\end{array}$ \\
\hline $\begin{array}{l}\text { Vance KW, Sansom SN, Lee S, } \\
\text { Chalei V, Kong L, Cooper SE, } \\
\text { Oliver PL, Ponting CP }\end{array}$ & 2014 & $\begin{array}{l}\text { The long non-coding } \\
\text { RNA Paupar regulates } \\
\text { the expression of both } \\
\text { local and distal genes } \\
\text { [CHART-seq] }\end{array}$ & $\begin{array}{l}\text { http://www.ncbi.nlm. } \\
\text { nih.gov/geo/query/ } \\
\text { acc.cgi?acc=GSE52571 }\end{array}$ & $\begin{array}{l}\text { Publicly available at } \\
\text { NCBI Gene Expression } \\
\text { Omnibus. }\end{array}$ \\
\hline
\end{tabular}

\section{References}

Bassett AR, Akhtar A, Barlow DP, Bird AP, Brockdorff N, Duboule D, Ephrussi A, Ferguson-Smith AC, Gingeras TR, Haerty W, Higgs DR, Miska EA, Ponting CP. 2014. Considerations when investigating IncRNA function in vivo. elife 3:e03058. doi: 10.7554/eLife.03058.

Berghoff EG, Clark MF, Chen S, Cajigas I, Leib DE, Kohtz JD. 2013. Evf2 (Dlx6as) IncRNA regulates ultraconserved enhancer methylation and the differential transcriptional control of adjacent genes. Development 140:4407-4416. doi: 10.1242/dev.099390.

Bhoopathi P, Chetty C, Dontula R, Gujrati M, Dinh DH, Rao JS, Lakka SS. 2011. SPARC stimulates neuronal differentiation of medulloblastoma cells via the Notch1/STAT3 pathway. Cancer Research 71:4908-4919. doi: 10.1158/0008-5472.CAN-10-3395.

Bros M, Boissel JP, Godtel-Armbrust U, Förstermann U. 2006. Transcription of human neuronal nitric oxide synthase mRNAs derived from different first exons is partly controlled by exon 1-specific promoter sequences. Genomics 87:463-473. doi: 10.1016/j.ygeno.2005.11.013.

Buske FA, Bauer DC, Mattick JS, Bailey TL. 2012. Triplexator: detecting nucleic acid triple helices in genomic and transcriptomic data. Genome Research 22:1372-1381. doi: 10.1101/gr.130237.111.

Cajanek L, Ribeiro D, Liste I, Parish CL, Bryja V, Arenas E. 2009. Wnt/beta-catenin signaling blockade promotes neuronal induction and dopaminergic differentiation in embryonic stem cells. Stem Cells 27:2917-2927. doi: 10.1002/stem.210.

Carvalho BS, Irizarry RA. 2010. A framework for oligonucleotide microarray preprocessing. Bioinformatics 26:2363-2367. doi: 10.1093/bioinformatics/btq431.

Chu C, Qu K, Zhong FL, Artandi SE, Chang HY. 2011. Genomic maps of long noncoding RNA occupancy reveal principles of RNA-chromatin interactions. Molecular Cell 44:667-678. doi: 10.1016/j.molcel.2011.08.027. 
Cusanovich DA, Pavlovic B, Pritchard JK, Gilad Y. 2014. The functional consequences of variation in transcription factor binding. PLOS Genetics 10:e1004226. doi: 10.1371/journal.pgen.1004226.

Di Ruscio A, Ebralidze AK, Benoukraf T, Amabile G, Goff LA, Terragni J, Figueroa ME, De Figueiredo Pontes LL, Alberich-Jorda M, Zhang P, Wu M, D'Alò F, Melnick A, Leone G, Ebralidze KK, Pradhan S, Rinn JL, Tenen DG. 2013. DNMT1-interacting RNAs block gene-specific DNA methylation. Nature 503:371-376. doi: 10.1038/ nature12598.

Dominguez MH, Ayoub AE, Rakic P. 2013. POU-III transcription factors (Brn1, Brn2, and Oct6) influence neurogenesis, molecular identity, and migratory destination of upper-layer cells of the cerebral cortex. Cerebral Cortex 23:2632-2643. doi: 10.1093/cercor/bhs252.

Eißmann M, Gutschner T, Hammerle M, Gunther S, Caudron-Herger M, Gross M, Schirmacher P, Rippe K, Braun T, Zörnig M, Diederichs S. 2012. Loss of the abundant nuclear non-coding RNA MALAT1 is compatible with life and development. RNA Biology 9:1076-1087. doi: 10.4161/rna.21089.

Fatica A, Bozzoni I. 2014. Long non-coding RNAs: new players in cell differentiation and development. Nature Reviews Genetics 15:7-21. doi: 10.1038/nrg3606.

Heger A, Webber C, Goodson M, Ponting CP, Lunter G. 2013. GAT: a simulation framework for testing the association of genomic intervals. Bioinformatics 29:2046-2048. doi: 10.1093/bioinformatics/btt343.

Hervouet E, Vallette FM, Cartron PF. 2010. Dnmt1/Transcription factor interactions: an alternative mechanism of DNA methylation inheritance. Genes \& Cancer 1:434-443. doi: 10.1177/1947601910373794.

Inomata K, Ohki I, Tochio H, Fujiwara K, Hiroaki H, Shirakawa M. 2008. Kinetic and thermodynamic evidence for flipping of a methyl-CpG binding domain on methylated DNA. Biochemistry 47:3266-3271. doi: 10.1021/bi7019029.

Kemp CR, Willems E, Wawrzak D, Hendrickx M, Agbor Agbor T, Leyns L. 2007. Expression of Frizzled5, Frizzled7, and Frizzled10 during early mouse development and interactions with canonical Wnt signaling. Developmental Dynamics 236:2011-2019. doi: 10.1002/dvdy.21198.

Laketa V, Simpson JC, Bechtel S, Wiemann S, Pepperkok R. 2007. High-content microscopy identifies new neurite outgrowth regulators. Molecular Biology of the Cell 18:242-252. doi: 10.1091/mbc.E06-08-0666.

Li LC, Dahiya R. 2002. MethPrimer: designing primers for methylation PCRs. Bioinformatics 18:1427-1431. doi: 10.1093/bioinformatics/18.11.1427.

Machanick P, Bailey TL. 2011. MEME-ChIP: motif analysis of large DNA datasets. Bioinformatics 27:1696-1697. doi: 10.1093/bioinformatics/btr189.

Martin D, Brun C, Remy E, Mouren P, Thieffry D, Jacq B. 2004. GOToolBox: functional analysis of gene datasets based on Gene Ontology. Genome Biology 5:R101. doi: 10.1186/gb-2004-5-12-r101.

Matys V, Kel-Margoulis OV, Fricke E, Liebich I, Land S, Barre-Dirrie A, Reuter I, Chekmenev D, Krull M, Hornischer K, Voss N, Stegmaier P, Lewicki-Potapov B, Saxel H, Kel AE, Wingender E. 2006. TRANSFAC and its module TRANSCompel: transcriptional gene regulation in eukaryotes. Nucleic Acids Research 34:D108-D110. doi: 10.1093/nar/gkj143.

McEvilly RJ, de Diaz MO, Schonemann MD, Hooshmand F, Rosenfeld MG. 2002. Transcriptional regulation of cortical neuron migration by POU domain factors. Science 295:1528-1532. doi: 10.1126/science.1067132.

McLean CY, Bristor D, Hiller M, Clarke SL, Schaar BT, Lowe CB, Wenger AM, Bejerano G. 2010. GREAT improves functional interpretation of cis-regulatory regions. Nature Biotechnology 28:495-501. doi: 10.1038/nbt.1630.

McLeay RC, Bailey TL. 2010. Motif Enrichment Analysis: a unified framework and an evaluation on ChIP data. BMC Bioinformatics 11:165. doi: 10.1186/1471-2105-11-165.

Melo CA, Drost J, Wijchers PJ, van de Werken H, de Wit E, Oude Vrielink JA, Elkon R, Melo SA, Leveille N, Kalluri R, de Laat W, Agami R. 2013. eRNAs are required for p53-dependent enhancer activity and gene transcription. Molecular Cell 49:524-535. doi: 10.1016/j.molcel.2012.11.021.

Ming GL, Song H. 2011. Adult neurogenesis in the mammalian brain: significant answers and significant questions. Neuron 70:687-702. doi: 10.1016/j.neuron.2011.05.001.

Mohammad F, Mondal T, Guseva N, Pandey GK, Kanduri C. 2010. Kenq1ot1 noncoding RNA mediates transcriptional gene silencing by interacting with Dnmt1. Development 137:2493-2499. doi: 10.1242/dev.048181.

Monnier P, Martinet C, Pontis J, Stancheva I, Ait-Si-Ali S, Dandolo L. 2013. H19 IncRNA controls gene expression of the Imprinted Gene Network by recruiting MBD1. Proceedings of the National Academy of Sciences of USA 110:20693-20698. doi: 10.1073/pnas.1310201110.

Mousavi K, Zare H, Dell'orso S, Grontved L, Gutierrez-Cruz G, Derfoul A, Hager GL, Sartorelli V. 2013. eRNAs promote transcription by establishing chromatin accessibility at defined genomic loci. Molecular Cell 51: 606-617. doi: 10.1016/j.molcel.2013.07.022.

Mutai H, Nagashima R, Sugitani Y, Noda T, Fujii M, Matsunaga T. 2009. Expression of Pou3f3/Brn-1 and its genomic methylation in developing auditory epithelium. Developmental Neurobiology 69:913-930. doi: 10.1002/dneu.20746.

Myers KA, Baas PW. 2007. Kinesin-5 regulates the growth of the axon by acting as a brake on its microtubule array. The Journal of Cell Biology 178:1081-1091. doi: 10.1083/jcb.200702074.

Nadar VC, Lin S, Baas PW. 2012. Microtubule redistribution in growth cones elicited by focal inactivation of kinesin-5. The Journal of Neuroscience 32:5783-5794. doi: 10.1523/JNEUROSCI.0144-12.2012.

Nakai S, Sugitani Y, Sato H, Ito S, Miura Y, Ogawa M, Nishi M, Jishage K, Minowa O, Noda T. 2003. Crucial roles of Brn1 in distal tubule formation and function in mouse kidney. Development 130:4751-4759. doi: 10.1242/dev.00666.

Ng SY, Bogu GK, Soh BS, Stanton LW. 2013. The long noncoding RNA RMST interacts with SOX2 to regulate neurogenesis. Molecular Cell 51:349-359. doi: 10.1016/j.molcel.2013.07.017.

Paoletti L, Elena C, Domizi P, Banchio C. 2011. Role of phosphatidylcholine during neuronal differentiation. IUBMB Life 63:714-720. doi: 10.1002/iub.521. 
Persengiev SP, Li J, Poulin ML, Kilpatrick DL. 2001. E2F2 converts reversibly differentiated PC12 cells to an irreversible, neurotrophin-dependent state. Oncogene 20:5124-5131. doi: 10.1038/sj.onc.1204663.

Ponjavic J, Oliver PL, Lunter G, Ponting CP. 2009. Genomic and transcriptional co-localization of protein-coding and long non-coding RNA pairs in the developing brain. PLoS genetics 5:e1000617. doi: 10.1371/journal.pgen.1000617.

Ramos AD, Diaz A, Nellore A, Delgado RN, Park KY, Gonzales-Roybal G, Oldham MC, Song JS, Lim DA. 2013. Integration of genome-wide approaches identifies IncRNAs of adult neural stem cells and their progeny in vivo. Cell Stem Cell 12:616-628. doi: 10.1016/j.stem.2013.03.003.

Rice P, Longden I, Bleasby A. 2000. EMBOSS: the european molecular biology Open software Suite. Trends in genetics 16:276-277. doi: 10.1016/S0168-9525(00)02024-2.

Sanjana NE, Cong L, Zhou Y, Cunniff MM, Feng G, Zhang F. 2012. A transcription activator-like effector toolbox for genome engineering. Nature Protocols 7:171-192. doi: 10.1038/nprot.2011.431.

Santoro F, Mayer D, Klement RM, Warczok KE, Stukalov A, Barlow DP, Pauler FM. 2013. Imprinted Igf2r silencing depends on continuous Airn IncRNA expression and is not restricted to a developmental window. Development 140:1184-1195. doi: 10.1242/dev.088849.

Sarker KP, Wilson SM, Bonni S. 2005. SnoN is a cell type-specific mediator of transforming growth factor-beta responses. The Journal of Biological Chemistry 280:13037-13046. doi: 10.1074/jbc.M409367200.

Sauvageau M, Goff LA, Lodato S, Bonev B, Groff AF, Gerhardinger C, Sanchez-Gomez DB, Hacisuleyman E, Li E, Spence M, Liapis SC, Mallard W, Morse M, Swerdel MR, D'Ecclessis MF, Moore JC, Lai V, Gong G, Yancopoulos GD, Frendewey D, Kellis M, Hart RP, Valenzuela DM, Arlotta P, Rinn JL. 2013. Multiple knockout mouse models reveal lincRNAs are required for life and brain development. eLife 2:e01749. doi: 10.7554/eLife.01749.

Shea TB, Fischer I, Sapirstein VS. 1985. Effect of retinoic acid on growth and morphological differentiation of mouse NB2a neuroblastoma cells in culture. Brain Research 353:307-314. doi: 10.1016/0165-3806(85)90220-2.

Shen Y, Yue F, McCleary DF, Ye Z, Edsall L, Kuan S, Wagner U, Dixon J, Lee L, Lobanenkov V, Ren B. 2012.

A map of the cis-regulatory sequences in the mouse genome. Nature 488:116-120. doi: 10.1038/nature11243. Simon MD. 2013. Capture hybridization analysis of RNA targets (CHART). In: Frederick MAusubel, et al, editors. Current protocols in molecular biology. Chapter 21, Unit 2125.

Simon MD, Wang Cl, Kharchenko PV, West JA, Chapman BA, Alekseyenko AA, Borowsky ML, Kuroda MI, Kingston RE. 2011. The genomic binding sites of a noncoding RNA. Proceedings of the National Academy of Sciences of USA 108:20497-20502. doi: 10.1073/pnas.1113536108.

Smyth GK. 2004. Linear models and empirical bayes methods for assessing differential expression in microarray experiments. Statistical applications in genetics and molecular biology 3:Article3.

Terashima M, Kobayashi M, Motomiya M, Inoue N, Yoshida T, Okano H, Iwasaki N, Minami A, Matsuoka I. 2010. Analysis of the expression and function of BRINP family genes during neuronal differentiation in mouse embryonic stem cell-derived neural stem cells. Journal of Neuroscience Research 88:1387-1393. doi: 10.1002/jnr.22315.

The ENCODE Project Consortium. 2012. An integrated encyclopedia of DNA elements in the human genome. Nature 489:57-74. doi: 10.1038/nature11247.

Tian D, Sun S, Lee JT. 2010. The long noncoding RNA, Jpx, is a molecular switch for $\mathrm{X}$ chromosome inactivation. Cell 143:390-403. doi: 10.1016/j.cell.2010.09.049.

Tremblay RG, Sikorska M, Sandhu JK, Lanthier P, Ribecco-Lutkiewicz M, Bani-Yaghoub M. 2010. Differentiation of mouse Neuro 2A cells into dopamine neurons. J. Neurosci. Methods 186:60-67. doi: 10.1016/j. jneumeth.2009.11.004.

Vallot C, Huret C, Lesecque Y, Resch A, Oudrhiri N, Bennaceur-Griscelli A, Duret L, Rougeulle C. 2013. XACT, a long noncoding transcript coating the active $\mathrm{X}$ chromosome in human pluripotent cells. Nature Genetics 45:239-241. doi: 10.1038/ng.2530.

Vance KW, Ponting CP. 2014. Transcriptional regulatory functions of nuclear long noncoding RNAs. Trends in Genetics 30:348-355. doi: 10.1016/j.tig.2014.06.001.

Vance KW, Sansom SN, Lee S, Chalei V, Kong L, Cooper SE, Oliver PL, Ponting CP. 2014. The long non-coding RNA Paupar regulates the expression of both local and distal genes. The EMBO Journal 33:296-311. doi: 10.1002/embj.201386225.

Visel A, Minovitsky S, Dubchak I, Pennacchio LA. 2007. VISTA Enhancer Browser-a database of tissue-specific human enhancers. Nucleic Acids Res 35 (Database issue):D88-D92. doi: 10.1093/nar/gkl822.

Wang KC, Yang YW, Liu B, Sanyal A, Corces-Zimmerman R, Chen Y, Lajoie BR, Protacio A, Flynn RA, Gupta RA, Wysocka J, Lei M, Dekker J, Helms JA, Chang HY. 2011. A long noncoding RNA maintains active chromatin to coordinate homeotic gene expression. Nature 472:120-124. doi: 10.1038/nature09819.

Watanabe K, Kamiya D, Nishiyama A, Katayama T, Nozaki S, Kawasaki H, Watanabe Y, Mizuseki K, Sasai Y. 2005. Directed differentiation of telencephalic precursors from embryonic stem cells. Nature Neuroscience 8: 288-296. doi: 10.1038/nn1402.

Xiong Z, Laird PW. 1997. COBRA: a sensitive and quantitative DNA methylation assay. Nucleic Acids Research 25:2532-2534. doi: 10.1093/nar/25.12.2532.

Yin T, Cook D, Lawrence M. 2012. ggbio: an R package for extending the grammar of graphics for genomic data. Genome Biology 13:R77. doi: 10.1186/gb-2012-13-8-r77.

Zhang B, Arun G, Mao YS, Lazar Z, Hung G, Bhattacharjee G, Xiao X, Booth CJ, Wu J, Zhang C, Spector DL. 2012. The IncRNA Malat1 is dispensable for mouse development but its transcription plays a cis-regulatory role in the adult. Cell Reports 2:111-123. doi: 10.1016/j.celrep.2012.06.003.

Zhang Y, Liu T, Meyer CA, Eeckhoute J, Johnson DS, Bernstein BE, Nusbaum C, Myers RM, Brown M, Li W, Liu XS. 2008. Model-based analysis of ChIP-Seq (MACS). Genome Biology 9:R137. doi: 10.1186/gb-2008-9-9-r137. 